\title{
A novel compressed sensing-based non-orthogonal multiple access scheme for massive MTC in 5G systems
}

\author{
Kesen $\mathrm{He}^{*}$ (D), Yangqing Li, Changchuan Yin and Yanbin Zhang
}

\begin{abstract}
The main challenges for massive machine type communication in $5 \mathrm{G}$ system are to support random access for massive users and to control signaling overhead and data processing complexity. To address these challenges, we propose a novel compressed sensing (CS)-based non-orthogonal multiple access (NOMA) scheme, called CS-NOMA, which introduces low coherence spreading (LCS) signatures to enable joint activity and data detection without requiring the activity information of users in advance. We present a sufficient condition for the construction of the LCS signatures to ensure that a CS-based multi-user detection (CS-MUD) can be effectively deployed in base station. Furthermore, we study the CS-NOMA scheme with imperfect channel state information (CSI) and present a bound for the performance of the CS-NOMA scheme. Simulation results show that the proposed scheme achieves a relatively high system overload (up to 4 ) when the active users are relatively sparse with an activity ratio of $1 \%$, which implies that the CS-NOMA scheme can significantly improve the spectral efficiency, avoid the control signaling overhead, and reduce the transmission latency.
\end{abstract}

Keywords: Non-orthogonal multiple access, Compressed sensing, Sparse multi-user detection, Massive machine type communication, Channel state information

\section{Introduction}

Massive machine type communication (MMTC) characterized by the requirements of low data rates, small packet sizes, and in some cases, tight delay constraints is expected to be one of the major drivers for the 5th generation (5G) wireless communication system. In the $5 \mathrm{G}$ system, a single base station will serve 10 to 100 times more machine type devices (MTDs) than the personal mobile phones, which poses great challenges to efficiently support massive users random access [1-3]. According to the statistics of mobile traffics [4], the number of active users is usually much smaller than the number of all possible users even in the busy hours in cellular communications, especially for 5G MMTC applications, where users can sporadically access or leave the system. Thus, the sparsity of user activity naturally exists in massive

${ }^{*}$ Correspondence: kesenhe@bupt.edu.cn

Beijing Laboratory of Advanced Information Networks, Beijing Key Laboratory of Network System Architecture and Convergence, Beijing University of Posts and Telecommunications, Beijing, China connectivity. In order to reduce the overhead of the MTD transmission, the sparse multi-user detection in the base station (BS) is expected to be deployed without requiring the activity knowledge of MTD, thereby reducing the control signaling overhead [5].

Recently, compressive sensing (CS) theory [6-8] has been widely used to recover sparse signals and also shows that reliable signal reconstruction far below the Nyquist sampling rate is possible provided that the signal is sparse. In order to address the challenge in future MMTC, the authors in [9-12] propose a novel physical layer signal processing scheme, called CS-based multi-user detection (CS-MUD), which takes advantage of the CS technology to detect the received sparse multi-user signals. The CS-MUD enables joint activity and data detection, which facilitates a reliable detection of direct random access. In $[9,13]$, the CS-MUD is deployed in a code division multiple access (CDMA) system. Then, a CS-MUD algorithm designed for single-carrier orthogonal frequency division multiplexing (SC-OFDM) systems is proposed in [10]. To improve the flexibility and scalability of accessing 
both time and frequency resources, Monsees et al. [11] and Bockelmann et al. [12] apply the CS-MUD to multicarrier transmissions. However, all these multiple access schemes belong to the category of orthogonal multiple access (OMA), which are difficult to meet the requirements of massive connectivity for MMTC in 5G systems.

To address the challenge of spectrum scarcity in 5G communications, recently some novel non-orthogonal multiple access (NOMA) schemes [14-20], e.g., the power domain NOMA [14], the low-density spreading (LDS) $[15,16]$, the sparse code multiple access (SCMA) [17], and the multi-user shared access (MUSA) [18], are proposed. In these well-known NOMA schemes, MUD is implemented using the successive interference cancellation (SIC) or the message passing algorithm (MPA), which requires the receiver to be exactly notified about the activity information of users in advance, resulting in hightransmission latency and control signaling overhead. In order to address this problem, the authors in [21, 22] proposed grant-free NOMA schemes for the OFDM system without requiring the activity information of users, which significantly reduces the signaling overhead and transmission latency.

However, nearly all the prior works on CS-based NOMA typically use the pseudo random noise (PN) sequence as the spread signatures and do not consider the general construction method for the spread signatures to ensure the sufficient recovery for users' signals in CSbased multi-user detection. In our recent work [23], we propose a novel CS-based NOMA scheme for the CDMA and /or the OFDM systems in which CS-MUD is deployed to enable joint activity and data detection without knowledge of the activity information of the users in advance. In this paper, we further extend the previous work to the more general case. We present a sufficient condition for the construction of the spreading signatures of users to ensure the successful CS-based multi-user detection. We also consider the case when the channel state information (CSI) is not perfect and present a bound on the performance of the CS-based NOMA scheme. In order to facilitate the understanding of different NOMA schemes in the literature, we compare them for the main different features in Table 1 . Note that in the table $|\mathbb{X}|$ denotes the cardinality of the constellation set $\mathbb{X}$, and $w$ is the maximum number of nonzero signals superimposed on each chip or subcarrier. In addition, $K, S$, and $N$ are the number of users, the number of active users, and the spreading factor, respectively.

We summarize the main contribution of this paper as follows:

1) We propose a CS-based NOMA scheme, called CSNOMA, and introduce a new version of spreading

Table 1 Comparison of different NOMA schemes

\begin{tabular}{|c|c|c|c|c|c|}
\hline $\begin{array}{l}\text { NOMA } \\
\text { scheme }\end{array}$ & $\begin{array}{l}\text { Detection } \\
\text { method }\end{array}$ & $\begin{array}{l}\text { Detection } \\
\text { complexity }\end{array}$ & $\begin{array}{l}\text { Spreading } \\
\text { sequence }\end{array}$ & $\begin{array}{l}\text { Scheduling } \\
\text { method }\end{array}$ & Key features \\
\hline $\begin{array}{l}\text { Power } \\
\text { domain } \\
\text { NOMA [14] }\end{array}$ & SIC & $\mathcal{O}\left(K^{3}\right)$ & $\mathrm{N} / \mathrm{A}$ & \multirow{4}{*}{$\begin{array}{l}\text { Dynamic } \\
\text { scheduling } \\
\text { to notify } \\
\text { the activity } \\
\text { information } \\
\text { of users. }\end{array}$} & \multirow{4}{*}{$\begin{array}{l}\text { High system } \\
\text { overload, high } \\
\text { transmission } \\
\text { latency and control } \\
\text { signaling overhead. }\end{array}$} \\
\hline LDS $[15,16]$ & MPA & $\mathcal{O}\left(|\mathbb{X}|^{w}\right)$ & $\begin{array}{l}\text { Sparse } \\
\text { spreading } \\
\text { sequence }\end{array}$ & & \\
\hline SCMA [17] & MPA & $\mathcal{O}\left(|\mathbb{X}|^{w}\right)$ & $\begin{array}{l}\text { Sparse } \\
\text { spreading } \\
\text { sequence }\end{array}$ & & \\
\hline MUSA [18] & SIC & $\mathcal{O}\left(K^{3}\right)$ & $\begin{array}{l}\text { Low } \\
\text { cross- } \\
\text { correlation } \\
\text { sequence }\end{array}$ & & \\
\hline $\begin{array}{l}\text { Grant-free } \\
\text { NOMA [22] }\end{array}$ & $\begin{array}{l}\text { Modified } \\
\text { OMP }\end{array}$ & $\mathcal{O}(S N K)$ & $\begin{array}{l}\text { PN } \\
\text { sequence }\end{array}$ & \multirow{2}{*}{$\begin{array}{l}\text { Grant-free } \\
\text { without the } \\
\text { knowledge } \\
\text { of activity } \\
\text { of users. }\end{array}$} & \multirow{2}{*}{$\begin{array}{l}\text { High system overload, } \\
\text { low transmission } \\
\text { latency, without control } \\
\text { signaling overhead. }\end{array}$} \\
\hline CS-NOMA & OMP & $\mathcal{O}(S N K)$ & $\begin{array}{l}\text { LCS sig- } \\
\text { nature }\end{array}$ & & \\
\hline
\end{tabular}


signature, called low coherence spreading (LCS) signature. We also present a sufficient condition for the construction of the LCS signatures and then theoretically prove the reliability of this condition.

2) We present a generating algorithm for the LCS matrix that consists of the LCS signatures of all users.

3) We present a bound on the performance of signal reconstruction under the case when the channel state information is not perfect.

The rest of the paper is structured as follows. In Section 2, we introduce the system model. In Section 3, we provide sufficient condition for the design of LCS signatures to ensure that CS-MUD can be effectively deployed in the $\mathrm{BS}$, and present the generating algorithm for the LCS matrix. In Section 4, we discuss the CS-NOMA scheme under imperfect CSI. In Section 5, we present the simulation results. Finally, we draw the conclusions in Section 6.

Notation: Throughout this paper, vectors and matrices will be represented by boldfaced lowercase and uppercase letters (e.g., $\mathbf{x}$ and $\mathbf{X}$ ), respectively. All vectors are defined as column vectors. Variables and constants are denoted in lowercase and uppercase letters (e.g., $x$ and $X$ ), respectively. Superscript $T$ and $\dagger$ represent the transpose and the Moore-Penrose pseudoinverse of a matrix, respectively. The notation $\mathcal{N}(0,1)$ is denoted as the Gaussian distribution with zero mean and unit variance, and $\mathbb{E}\{x\}$ represents the mean of $x$.

\section{System model}

We consider an MMTC scenario where a set of users (i.e., MTDs), $\mathcal{K}=\{1, \ldots, K\}$, sporadically accesses to a single BS over multi-path wireless channels in the presence of additive white Gaussian noise (AWGN). All users share $N$ $(N \ll K)$ CDMA chips at the same time, which means that the gain of the spreading signature is $N$. Furthermore, we consider that at most $S(S \ll N)$ users are active in a given time, and let $\rho_{\mathrm{a}}=S / K\left(\rho_{\mathrm{a}} \ll 1\right)$ and $\beta=K / N(\beta>1)$ denote the activity ratio and the system overload, respectively. We assume that the active users transmit symbols from a symbol alphabet $\mathcal{A}$, and the inactive users transmit nothing, i.e., the transmitted symbols are equal to zero. In the following, we present the proposed CS-NOMA scheme for the CDMA system.

In a conventional CDMA system, user separation can be achieved by uniquely assigning orthogonal spreading signature to each user. However, given the gain $N$, the number of orthogonal spreading signatures cannot exceed $N$, which means that the total number of users that the system can support at the same time cannot be greater than $N$. In our proposed CS-NOMA scheme shown in Fig. 1, we introduce a new version of spreading signature, called low coherence spreading (LCS) signature, to achieve the system overload. Unlike orthogonal spreading signatures whose mutual coherence is equal to zero, the mutual coherence between the LCS signatures is nonzero, but is very low, such as far less than 1 . Specifically, given the gain $N$, the number of LCS signatures can exceed $N$, resulting in a system overload, i.e., $\beta>1$. Let $x_{k} \in \mathbb{X}$ be the transmitted symbol of user $k \in \mathcal{K}$, where $\mathbb{X}=$ $\{\mathcal{A} \cup 0\}$, then let $\mathbf{x}=\left[x_{1}, \ldots, x_{K}\right]^{\top} \in \mathbb{X}^{K \times 1}$ be the multiuser transmitted signal vector. We assume that at most $S$ users are active at a given time, which means that up to $S$ users transmit symbols while other users transmit nothing at a given time. Thus, based on the CS theory [6], the multi-user transmitted signal vector $\mathbf{x}$ is an $S$ sparse signal vector, i.e., there are at most $S$ non-zero elements in $\mathbf{x}$. Symbol $x_{k}$ spreads over the LCS signature $\mathbf{f}_{k}=\left[f_{1, k}, \ldots, f_{N, k}\right]^{\top} \in \mathbb{R}^{N \times 1}$ for user $k$. Then, the LCS matrix is defined by $\mathcal{F}=\left[\mathbf{f}_{1}, \ldots, \mathbf{f}_{K}\right] \in \mathbb{R}^{N \times K}$, which contains the LCS signatures of all users.

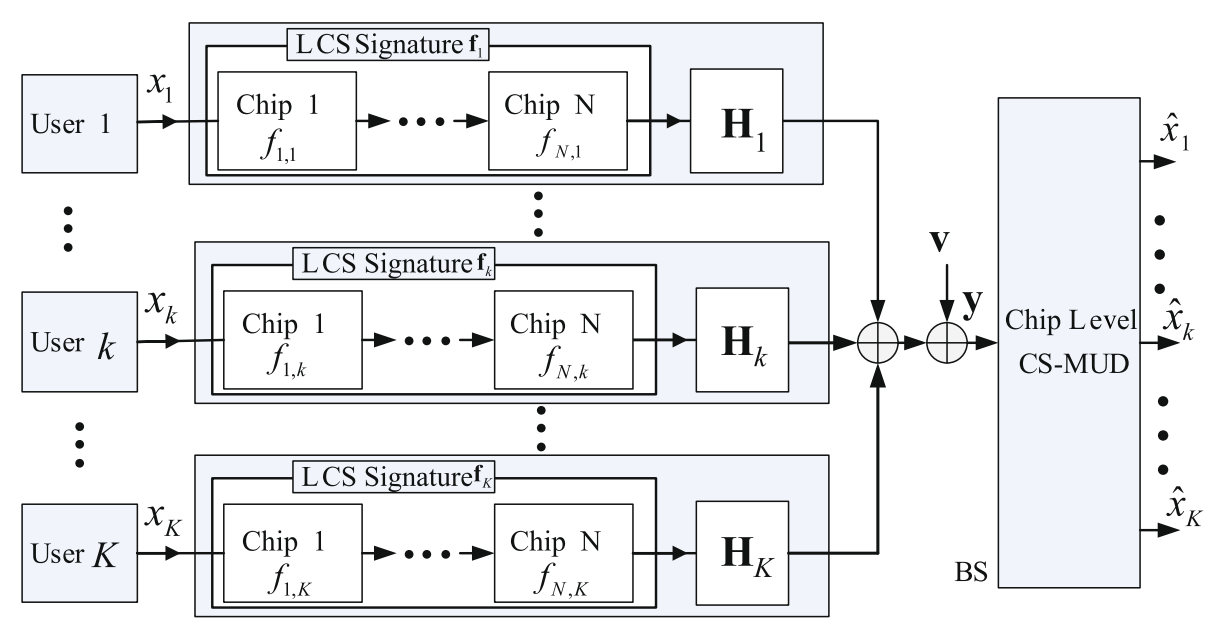

Fig. 1 The CS-NOMA scheme for the CDMA system 
The proposed CS-NOMA scheme is essentially a typical asynchronous CDMA uplink transmission scheme similar to [24]. Furthermore, the spread symbols of each user are distorted by a user-specific frequency-selective channel with impulse response $\mathbf{h}_{k}=\left[h_{1, k}, \ldots, h_{L, k}\right]^{\top} \in \mathbb{C}^{L \times 1}$ $(k \in \mathcal{K})$ of length $L$ which is constant for a whole frame, and let $\mathcal{H}=\left[\mathbf{h}_{1}, \ldots, \mathbf{h}_{K}\right] \in \mathbb{C}^{L \times K}$ denote the channel response matrix of all users with the same number of channel paths $L$. Then, considering the effect of convolution operation of the channels on users' signals, we can model the channel matrix for user $k$ as

$$
\mathbf{H}_{k}=\left[\begin{array}{ccccc}
h_{1, k} & & & & \\
h_{2, k} & h_{1, k} & & & \\
\vdots & h_{2, k} & \ddots & & \\
h_{L, k} & \vdots & & \ddots & \\
& h_{L, k} & & h_{1, k} & \\
& & & h_{2, k} & h_{1, k}
\end{array}\right]_{N \times N}
$$

where the values of the entries which are not shown in the matrix $\mathbf{H}_{k}$ in (1) are zeros, and such typical channel model has been widely used in machine-type communication (MTC) $[9,13]$. In the BS, the received signal formed by $N$ chips is denoted by $\mathbf{y}=\left[y_{1}, \ldots, y_{N}\right]^{\top} \in \mathbb{C}^{N \times 1}$, and the noise vector is defined as $\mathbf{v}=\left[v_{1}, \ldots, v_{N}\right]^{\top} \in \mathbb{C}^{N \times 1}$. Thus, the received signal vector is given by

$$
\begin{aligned}
\mathbf{y} & =\sum_{k=1}^{K} \mathbf{H}_{k} \mathbf{f}_{k} x_{k}+\mathbf{v} \\
& =\mathbf{B x}+\mathbf{v},
\end{aligned}
$$

where the effective channel matrix $\mathbf{B}$ can be written as

$$
\mathbf{B}=\left[\mathbf{H}_{1} \mathbf{f}_{1}, \ldots, \mathbf{H}_{K} \mathbf{f}_{K}\right] \in \mathbb{C}^{N \times K} .
$$

In order to enable joint activity and data detection without requiring the activity knowledge of users, the CSMUD is deployed in the BS to recover the sparse signal $\mathbf{x}$ in (3). In Fig. $1, \hat{\mathbf{x}}=\left[\hat{x}_{1}, \ldots, \hat{x}_{K}\right]^{\top} \in \mathbb{X}^{K \times 1}$ is the estimation of $\mathbf{x}$; and it can be achieved by the orthogonal matching pursuit (OMP) algorithm [25, 26], which is a typical greed algorithm for CS-MUD. Based on the CS theory [6, 7], the effective channel matrix $\mathbf{B}$ in (3) is expected to be an effective sensing matrix which guarantees the recovery of the sparse signal $\mathbf{x}$. In (4), B is a synthetic matrix generated by the channel matrices (i.e., $\mathbf{H}_{1}, \ldots, \mathbf{H}_{K}$ ) and the LCS signatures (i.e., $\mathbf{f}_{1}, \ldots, \mathbf{f}_{K}$ ) of all users. Specifically, due to the physical propagation property of the channel, the channel matrices naturally exist in some form and can not be artificially controlled. Thus, we expect that the effective channel matrix $\mathbf{B}$ is an effective sensing matrix. Therefore, in the next section, we will discuss how to design the LCS signatures (i.e., the design of $\mathcal{F}$ ) to ensure that the effective channel matrix $\mathbf{B}$ guarantees the recovery of the sparse signal $\mathbf{x}$.

\section{Design of the LCS signatures}

In this section, we discuss how to design the LCS signatures to ensure that the effective channel matrix B in (4) to be an effective sensing matrix which can guarantee the recovery of the sparse signal $\mathbf{x}$. According to the CS theory $[27,28]$, coherence of the sensing matrix (i.e., B) can provide sufficient condition for guaranteeing recovery of the sparse signal. Therefore, by analyzing the coherence of matrix B, we present sufficient conditions at which the LCS signatures should satisfy. Then, based on these conditions for the design of the LCS signatures, we present a generating algorithm to construct the LCS matrix $\mathcal{F}$. Note that in this section we only consider the design of LCS signatures and the effective channel matrix in real number value, which can be extended directly to the complex number value case.

\subsection{Coherence of the effective channel matrix}

Now, we first give the definition of the mutual coherence between two nonzero vectors $\mathbf{a}_{1}, \mathbf{a}_{2} \in \mathbb{R}^{N \times 1}$, i.e.,

$$
\mu\left(\mathbf{a}_{1}, \mathbf{a}_{2}\right)=\frac{\left|\left\langle\mathbf{a}_{1}, \mathbf{a}_{2}\right\rangle\right|}{\left\|\mathbf{a}_{1}\right\|_{2}\left\|\mathbf{a}_{2}\right\|_{2}},
$$

where $\left\langle\mathbf{a}_{1}, \mathbf{a}_{2}\right\rangle$ denotes the inner product between $\mathbf{a}_{1}$ and $\mathbf{a}_{2}$, i.e., $\left\langle\mathbf{a}_{1}, \mathbf{a}_{2}\right\rangle=\mathbf{a}_{1}^{\top} \mathbf{a}_{2}$. Then, the coherence of $\mathbf{A}=$ $\left[\mathbf{a}_{1}, \ldots, \mathbf{a}_{K}\right] \in \mathbb{R}^{N \times K}$, denoted by $\mu(\mathbf{A})$, is defined as [28-31]

$$
\mu(\mathbf{A})=\max _{i \neq j} \mu\left(\mathbf{a}_{i}, \mathbf{a}_{j}\right) .
$$

It can be shown that $\mu(\mathbf{A}) \in\left[\sqrt{\frac{K-N}{N(K-1)}}, 1\right][32,33]$. Note that when $K \gg N$, the lower bound, known as the Welch bound [32], is approximately $\mu(\mathbf{A}) \geq 1 / \sqrt{N}$.

In order to facilitate analysis, we rewrite (4) as

$$
\mathbf{B}=\left[\mathbf{F}_{1} \mathbf{h}_{1}, \ldots, \mathbf{F}_{K} \mathbf{h}_{K}\right] \in \mathbb{R}^{N \times K},
$$

where $\mathbf{F}_{k} \in \mathbb{R}^{N \times L}(1 \leq k \leq K)$ is generated by $\mathbf{f}_{k}$ in the following form, i.e.,

$$
\mathbf{F}_{k}=\left[\begin{array}{ccccc}
f_{1, k} & 0 & & \\
f_{2, k} & f_{1, k} & 0 & \\
f_{3, k} & f_{2, k} & \ddots & \\
\vdots & \vdots & & f_{1, k} \\
\vdots & \vdots & & \vdots \\
f_{N, k} & f_{N-1, k} & \cdots & f_{N-L+1, k}
\end{array}\right]_{N \times L} .
$$

Then, we denote the inner product between the $m$ th column of $\mathbf{F}_{k}$ and the $n$th column of $\mathbf{F}_{j}(1 \leq m, n \leq L, 1 \leq$ $k, j \leq K)$ as

$$
\mu_{m, n}^{k j}=\left\langle\mathbf{F}_{k}(:, m), \mathbf{F}_{j}(:, n)\right\rangle,
$$

where the notation $\mathbf{A}(:, t)$ represents the $t$ th column of $\mathbf{A}$. 
In the sequel, we first present a theorem to clarify the conditions at which the LCS signatures should satisfy to ensure the sparse signal $\mathbf{x}$ can be recovered, then based on this theorem, we propose a construction algorithm for the LCS matrix F in Section 3.2.

Theorem 1 Given two matrices $\mathcal{H}=\left[\mathbf{h}_{1}, \ldots, \mathbf{h}_{K}\right] \in$ $\mathbb{R}^{L \times K}$ and $\mathcal{F}=\left[\mathbf{f}_{1}, \ldots, \mathbf{f}_{K}\right] \in \mathbb{R}^{N \times K}, 2<L<N . \mathcal{F}$ has unit-norm columns, i.e., $\left\|\mathbf{f}_{k}\right\|_{2}^{2}=1$, and $\mathbf{h}_{k} \neq \mathbf{0}$, $1 \leq k \leq K . \mathbf{F}_{k} \in \mathbb{R}^{N \times L}$ is full rank and generated by $\mathbf{f}_{k}$ in (8). If there exist constants $\mu_{1}, \mu_{2}, \theta \in[0,1)$ satisfying the following properties for $1 \leq m, n \leq L$ and $1 \leq k, j \leq K$ :

$$
\begin{aligned}
& \text { - } \sum_{n=1, n \neq m}^{L}\left|\mu_{m, n}^{k k}\right| \leq \mu_{1}, \forall m, k ; \\
& \text { - } \sum_{m=1}^{L}\left|\mu_{m, n}^{k j}\right| \leq \mu_{2}, k \neq j, \forall n, k, j ; \\
& \text { - } \sum_{l=1}^{L-1} f_{N-l+1, k}^{2} \leq \theta,
\end{aligned}
$$

then, for arbitrary $\theta+\mu_{1}<1$, the coherence of $\mathbf{B}=$ $\left[\mathbf{F}_{1} \mathbf{h}_{1}, \ldots, \mathbf{F}_{K} \mathbf{h}_{K}\right] \in \mathbb{R}^{N \times K}$, i.e., $\mu(\mathbf{B})$, satisfies the following inequality

$$
\mu(\mathbf{B}) \leq \frac{\mu_{2}}{1-\theta-\mu_{1}} .
$$

The proof of Theorem 1 is presented in Appendix 1.

From Theorem 1, we have an upper bound for the coherence of $\mathbf{B}$, which is denoted as

$$
\mu_{\text {up }}=\frac{\mu_{2}}{1-\theta-\mu_{1}} \text {. }
$$

Moreover, from the proof of Theorem 1 in Appendix 1, we have that the upper bound $\mu_{\text {up }}$ is only determined by the LCS signatures $\mathbf{f}_{1}, \ldots, \mathbf{f}_{K}$ (i.e., matrix $\mathcal{F}$ ) and has nothing to do with the channel response vectors $\mathbf{h}_{1}, \ldots, \mathbf{h}_{K}$ (i.e., matrix $\mathcal{H}$ ). In [27, 28], the authors have proved that the coherence of a sensing matrix $\mathbf{B}$ (i.e., $\mu(\mathbf{B}))$ can guarantee the recovery of $S$-sparse signal when $S$ satisfies

$$
S<\frac{1}{2}\left(1+\frac{1}{\mu(\mathbf{B})}\right)
$$

Based on Theorem 1, the LCS signatures satisfying the properties (10)-(12) ensure that $\mu(\mathbf{B}) \leq \mu_{\text {up }}=\frac{\mu_{2}}{1-\theta-\mu_{1}}$, so we have the minimum sparsity determined by $B$ as follows

$$
S_{\min }=\frac{1}{2}\left(1+\frac{1}{\mu_{\mathrm{up}}}\right) \leq \frac{1}{2}\left(1+\frac{1}{\mu(\mathbf{B})}\right) .
$$

From (15), we have that when $S<S_{\min }$, the effective channel matrix $\mathbf{B}$ absolutely guarantees the recovery of the $S$-sparse signal $\mathbf{x}$.
In summary, Theorem 1 presents a sufficient condition for the construction of the LCS signatures. Specifically, when the LCS signatures or the matrix $\mathcal{F}$ satisfy the properties in (10)-(12), based on the above discussion, we can ensure that the effective channel matrix $\mathbf{B}$ in (3) is an effective sensing matrix which can guarantee the recovery of $S_{\text {min }}$-sparse signal $\mathbf{x}$.

In order to further interpret the proposed CS-NOMA scheme with the LCS signatures satisfying Theorem 1, we introduce the restricted isometry property (RIP)[7] which is another necessary and sufficient condition for guaranteeing the recovery of the sparse signal $\mathbf{x}$ in the presence of noise [7, 27]. First, we give the definition of RIP, i.e., a matrix A satisfies the RIP of order $S$ if there exists a $\delta_{S} \in(0,1)$ such that

$$
\left(1-\delta_{S}\right)\|\mathbf{x}\|_{2}^{2} \leq\|\mathbf{A} \mathbf{x}\|_{2}^{2} \leq\left(1+\delta_{S}\right)\|\mathbf{x}\|_{2}^{2}
$$

holds for all $S$-sparse signal $\mathbf{x}$. In the following, we present a corollary of Theorem 1, which analyzes the RIP of $\mathbf{B}$ consisting of $\mathcal{H}$ with unit-norm columns.

Corollary 1 In Theorem 1 , when $\mathcal{H}$ has unit-norm columns, i.e., $\left\|\mathbf{h}_{k}\right\|_{2}^{2}=1,1 \leq k \leq K$, then $\mathbf{B}$ has the RIP of order $S$ with constant $\delta_{S}=\theta+\mu_{1}+(S-1) \mu_{2}$ for arbitrary $S<1+1 / \mu_{\text {up }}$, i.e.,

$$
\left(1-\delta_{S}\right)\|\mathbf{x}\|_{2}^{2} \leq\|\mathbf{B} \mathbf{x}\|_{2}^{2}<\left(1+\delta_{S}\right)\|\mathbf{x}\|_{2}^{2},
$$

where $\mathbf{x} \in \mathbb{R}^{K \times 1}$ is an S-sparse signal.

The proof of Corollary 1 is presented in Appendix 2 .

In this paper, we assume that each user has the same transmit power and then normalize the channel response vectors of all users $\mathbf{h}_{1}, \ldots, \mathbf{h}_{K}$, then the channel response matrix $\mathcal{H}$ will have unit-columns. In addition, the authors of $[34,35]$ have proved that a matrix A satisfying the RIP of order $2 S$ can guarantee the recovery of the $S$-sparse signal. Thus, in our CS-NOMA scheme, from Corollary 1, the LCS signatures satisfying Theorem 1 ensure that $\mathbf{B}$ has the RIP of order $2 S<2 S_{\min }=1+1 / \mu_{\text {up }}$ which guarantees the recovery of $S$-sparse signal in the presence of noise.

Based on the above analysis, the LCS signatures satisfying Theorem 1 can guarantee the recovery of a sparse signal either in terms of the coherence property or RIP. In the following, we present a generating algorithm for the LCS matrix $\mathcal{F}$ based on Theorem 1.

\subsection{Generating algorithm of the LCS matrix $\mathcal{F}$}

Based on the results in Theorem 1, the LCS matrix $\mathcal{F} \in$ $\mathbb{R}^{N \times K}$ can be obtained by system searching over the set $\{\xi\}$ by using Algorithm 1, where the entries of $\xi \in \mathbb{R}^{N \times 1}$ are independent realizations of random variables with 
following distribution

$$
\varsigma_{n}:=\left\{\begin{array}{cc}
+\frac{1}{\sqrt{M}}, & \text { with probability } \frac{M}{2 N} \\
0, & \text { with probability } \frac{N-M}{N} \\
-\frac{1}{\sqrt{M}}, & \text { with probability } \frac{M}{2 N},
\end{array}\right.
$$

where $\varsigma_{n}$ is the $n$th entry of $\xi$. In this paper, we consider $N=M$, which implies that the entries of $\xi$ are independent realizations of \pm Bernoulli random variables with

$$
\varsigma_{n}:= \begin{cases}+\frac{1}{\sqrt{M}}, & \text { with probability } \frac{1}{2} \\ -\frac{1}{\sqrt{M}}, & \text { with probability } \frac{1}{2} .\end{cases}
$$

Thus, from Theorem 1 , we have $\theta=L / M$. In the following, we give some interpretation on Algorithm 1.

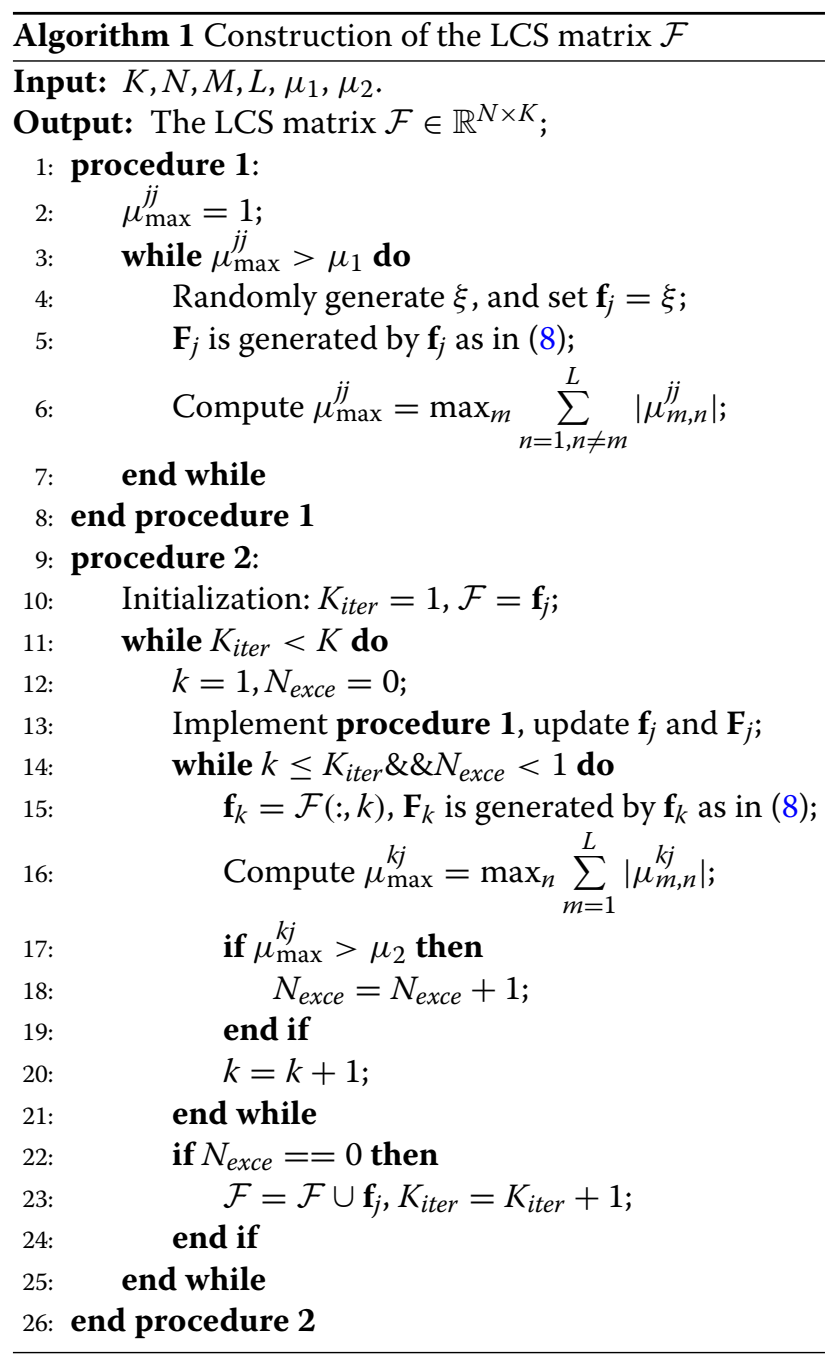

In Algorithm 1, procedure 1 and procedure 2 ensure that the designed LCS signatures satisfy the properties in (10) and (11), respectively. In procedure 1, we randomly generate $\xi$ and let $\mathbf{f}_{j}=\xi$, repeating this process until finding a solution of $\xi$ that ensures $\mu_{\max }^{j j}=\max _{m} \sum_{n=1, n \neq m}^{L}\left|\mu_{m, n}^{j j}\right| \leq$ $\mu_{1}$, so the property in (10) is satisfied. In procedure 2 , the LCS matrix $\mathcal{F}$ is initialized as $\mathbf{f}_{j}$ obtained in procedure 1. $K_{i t e r}$ denotes the number of the columns of $\mathcal{F}$ and is initialized as $1 . N_{\text {exce }}$ is defined as the number of the columns of $\mathcal{F}$ that makes $\mu_{\max }^{k j}=\max _{n} \sum_{m=1}^{L}\left|\mu_{m, n}^{k j}\right|>\mu_{2}$ with $\mathbf{f}_{j}$ and is initialized as 0 in each repeat of the while at line 11. Then, $\mathbf{f}_{j}$ and $\mathbf{F}_{j}$ are updated by implementing procedure 1 . We compute the $\mu_{\max }^{k j}$ between $\mathbf{f}_{k}\left(1 \leq k \leq K_{i t e r}\right)$ and $\mathbf{f}_{j}$. If $\mu_{\max }^{k j}>\mu_{2}$, i.e., $N_{\text {exce }}=1$, we stop the while at line 14 and then repeat the while at line 11. If $\mu_{\max }^{k j} \leq \mu_{2}$ for arbitrary $k$, i.e., $N_{\text {exce }}=0$, we update $\mathcal{F}=\mathcal{F} \cup \mathbf{f}_{\mathbf{j}}, K_{\text {iter }}=K_{\text {iter }}+1$, and then repeat the while at line 11 . We repeat the above operations until $K=K_{i t e r}$, and finally output the LCS matrix $\mathcal{F}$.

\section{Effect of the imperfect channel state information (CSI)}

Since the CS-MUD is applied to perform joint activity and data detection, the BS needs to know the sensing matrix $\mathbf{B}=\left[\mathbf{F}_{1} \mathbf{h}_{1}, \ldots, \mathbf{F}_{K} \mathbf{h}_{K}\right]$ based on the CS theory [7]. Because the LCS matrix $\mathcal{F}$ is designed in advance, i.e., $\mathbf{F}_{k}(1 \leq k \leq K)$ is known to the BS. The channel response vector $\mathbf{h}_{k}(1 \leq k \leq K)$ is generally obtained by using a channel estimation algorithm in BS. However, the channel state information (CSI) is generally not perfect, which means that there exists some error in the estimation of $\mathbf{h}_{k}$ in BS. Let $\hat{\mathbf{h}}_{k}$ and $\mathbf{e}_{k}$ denote the estimated channel response vector and the error vector of user $k$, respectively. Then, we have

$$
\hat{\mathbf{h}}_{k}=\mathbf{h}_{k}+\mathbf{e}_{k}, \forall k
$$

Correspondingly, let $\hat{\mathbf{B}}=\left[\mathbf{F}_{1} \hat{\mathbf{h}}_{1}, \ldots, \mathbf{F}_{K} \hat{\mathbf{h}}_{K}\right]$ denote the estimated matrix of $\mathbf{B}$ in the BS. From Theorem 1, we know that the upper bound of $\mu(\mathbf{B})$, i.e., $\mu_{\text {up }}$, is only determined by the LCS signatures $\mathbf{f}_{1}, \ldots, \mathbf{f}_{K}$ (i.e., the LCS matrix $\mathcal{F}$ ) and has nothing to do with the channel response vectors $\mathbf{h}_{1}, \ldots, \mathbf{h}_{K}$ (i.e., the channel response matrix $\mathcal{H}$ ). Specifically, the differences between $\mathbf{B}$ and $\hat{\mathbf{B}}$ only exist in the channel response vectors, i.e., $\mathbf{B}$ and $\hat{\mathbf{B}}$ have the same upper bound of coherence $\mu_{\mathrm{up}}$. Then, the coherence of $\hat{\mathbf{B}}$, i.e., $\mu(\hat{\mathbf{B}})$, satisfies the inequality

$$
\mu(\hat{\mathbf{B}}) \leq \mu_{\text {up }}=\frac{\mu_{2}}{1-\theta-\mu_{1}},
$$

which implies that $\hat{\mathbf{B}}$ is also an effective sensing matrix.

In the sequel, we analyze the performance of signal reconstruction under the imperfect CSI case for the CS-NOMA scheme. We first introduce Theorem 2 which plays an important role in analysis of the performance. 
Theorem 2 (Theorem 3.1 in [36]). Suppose that the coherence of $\mathbf{A} \in \mathbb{R}^{N \times K}(N \ll K)$ is $\mu_{\mathbf{A}}$ and $\mathbf{x} \in \mathbb{R}^{K \times 1}$ is an $S$-sparse signal, where $S<\left(1 / \mu_{\mathbf{A}}+1\right) / 4$. Furthermore, suppose that the measurement $\mathbf{y} \in \mathbb{R}^{N \times 1}$ is obtained by $\mathbf{y}=\mathbf{A x}+\mathbf{n}$, where $\mathbf{n} \in \mathbb{R}^{N \times 1}$ is a noise vector. Then, the estimation of $\mathbf{x}$ is given by

$$
\hat{\mathbf{x}}=\underset{\mathbf{z}}{\arg \min }\|\mathbf{z}\|_{1} \text {, subject to } \quad \mathbf{z} \in \mathfrak{B}(\mathbf{y}),
$$

where $\mathfrak{B}(\mathbf{y})=\left\{\mathbf{z}:\|\mathbf{A z}-\mathbf{y}\|_{2} \leq \epsilon\right\}$. Then, we have that

$$
\|\mathbf{x}-\hat{\mathbf{x}}\|_{2} \leq \frac{\|\mathbf{n}\|_{2}+\epsilon}{\sqrt{1-\mu_{\mathbf{A}}(4 S-1)}} .
$$

Theorem 2 provides a bound for the worst-case performance given a bounded noise $\mathbf{n}$, i.e., $\|\mathbf{n}\|_{2} \leq C$, where $C$ is an absolute constant $[36,37]$. Note that this theorem holds for the case when $\epsilon=0$ as well as $\|\mathbf{n}\|_{2}=0$. Thus, it also applies to the noise-free setting. Furthermore, there is no restriction on $\|\mathbf{n}\|_{2} \leq \epsilon$. In fact, this theorem is valid even when $\epsilon=0$ but $\|\mathbf{n}\|_{2} \neq 0$ [37]. However, as noted in [36], Theorem 2 is the result of a worst-case analysis and will typically overestimate the actual error.

In the perfect CSI case, from Theorem 2, the CSNOMA scheme has a coherence-based bound on the performance for the bounded noise $\mathbf{v}$, which is given by

$$
\|\mathbf{x}-\hat{\mathbf{x}}\|_{2} \leq \frac{\|\mathbf{v}\|_{2}+\epsilon}{\sqrt{1-\mu_{\mathbf{B}}(4 S-1)}} \leq \frac{\|\mathbf{v}\|_{2}+\epsilon}{\sqrt{1-\mu_{\text {up }}(4 S-1)}},
$$

where $\mu_{\mathbf{B}}$ is the coherence of $\mathbf{B}$, and $S<\left(1 / \mu_{\text {up }}+1\right) / 4$. In this paper, we denote the perfect LCS signatures as ones that are designed with the parameters $\mu_{1} \rightarrow 0, \mu_{2} \rightarrow$ 0 , and $\theta \neq 1$ (i.e., $\mu_{\mathbf{B}} \rightarrow 0$ ). From (25), the CS-NOMA scheme using the perfect LCS signatures has a bound, i.e.,

$$
\|\mathbf{x}-\hat{\mathbf{x}}\|_{2} \leq\|\mathbf{v}\|_{2}+\epsilon \text {. }
$$

In fact, the channel response estimation error can eventually be considered as a noise error. Thus, based on Theorem 2, we analyze the bound on the performance of the CS-NOMA scheme under imperfect CSI for bounded $\mathbf{e}_{k}, 1 \leq k \leq K$. For the corresponding $\hat{\mathbf{B}}=\left[\mathbf{F}_{1} \hat{\mathbf{h}}_{1}, \ldots, \mathbf{F}_{K} \hat{\mathbf{h}}_{K}\right]$, we rewrite it as $\hat{\mathbf{B}}=$ $\left[\mathbf{F}_{1} \mathbf{h}_{1}+\mathbf{F}_{1} \mathbf{e}_{1}, \ldots, \mathbf{F}_{K} \mathbf{h}_{K}+\mathbf{F}_{K} \mathbf{e}_{K}\right]$. Thus, the received signal in (3) is rewritten as

$$
\begin{aligned}
\mathbf{y} & =\hat{\mathbf{B}} \mathbf{x}+\mathbf{v} \\
& =\mathbf{B} \mathbf{x}+\mathbf{B}_{\mathbf{e}} \mathbf{x}+\mathbf{v},
\end{aligned}
$$

where $\mathbf{B}_{\mathbf{e}}=\left[\mathbf{F}_{1} \mathbf{e}_{1}, \ldots, \mathbf{F}_{K} \mathbf{e}_{K}\right]$. Let $\mathbf{n}=\mathbf{B}_{\mathbf{e}} \mathbf{x}+\mathbf{v}$ denotes the total noise. From Theorem 2, when $\mu(\mathbf{B})$ and $S$ are fixed, the upper bound of $\|\mathbf{x}-\hat{\mathbf{x}}\|_{2}$ is determined by the upper bound of $\|\mathbf{n}\|_{2}$, i.e., $\left\|\mathbf{B}_{\mathbf{e}} \mathbf{x}+\mathbf{v}\right\|_{2}$. Thus, based on the triangle inequality [38], we have

$$
\|\mathbf{n}\|_{2}=\left\|\mathbf{B}_{\mathbf{e}} \mathbf{x}+\mathbf{v}\right\|_{2} \leq\left\|\mathbf{B}_{\mathbf{e}} \mathbf{x}\right\|_{2}+\|\mathbf{v}\|_{2} .
$$

In order to obtain an upper bound for $\|\mathbf{n}\|_{2}$, we need to analyze the upper bound of $\left\|\mathbf{B}_{\mathbf{e}} \mathbf{x}\right\|_{2}$. Similar to (46) in Appendix 1, the bound of $\left\|\mathbf{B}_{\mathbf{e}} \mathbf{x}\right\|_{2}^{2}$ is given by

$$
\lambda_{\min }^{\mathbf{D}}\|\mathbf{x}\|_{2}^{2} \leq\left\|\mathbf{B}_{\mathbf{e}} \mathbf{x}\right\|_{2}^{2} \leq \lambda_{\text {max }}^{\mathbf{D}}\|\mathbf{x}\|_{2}^{2},
$$

where $\lambda_{\min }^{\mathbf{D}}$ and $\lambda_{\max }^{\mathbf{D}}$ are the minimum and maximum eigenvalues of $\mathbf{D}=\mathbf{B}_{\mathbf{e}}^{\top} \mathbf{B}_{\mathbf{e}} \in \mathbb{R}^{K \times K}$, respectively. $\mathbf{D}$ is represented by

$$
\left[\begin{array}{cccc}
\left(\mathbf{F}_{1} \mathbf{e}_{1}\right)^{T} \mathbf{F}_{1} \mathbf{e}_{1} & \left(\mathbf{F}_{1} \mathbf{e}_{1}\right)^{T} \mathbf{F}_{2} \mathbf{e}_{2} & \cdots & \left(\mathbf{F}_{1} \mathbf{e}_{1}\right)^{T} \mathbf{F}_{K} \mathbf{e}_{K} \\
\left(\mathbf{F}_{2} \mathbf{e}_{2}\right)^{T} \mathbf{F}_{1} \mathbf{e}_{1} & \left(\mathbf{F}_{2} \mathbf{e}_{2}\right)^{T} \mathbf{F}_{2} \mathbf{e}_{2} & \cdots & \left(\mathbf{F}_{2} \mathbf{e}_{2}\right)^{T} \mathbf{F}_{K} \mathbf{e}_{K} \\
\vdots & \vdots & \ddots & \vdots \\
\left(\mathbf{F}_{K} \mathbf{e}_{K}\right)^{T} \mathbf{F}_{1} \mathbf{e}_{1} & \left(\mathbf{F}_{K} \mathbf{e}_{K}\right)^{T} \mathbf{F}_{2} \mathbf{e}_{2} & \cdots & \left(\mathbf{F}_{K} \mathbf{e}_{K}\right)^{T} \mathbf{F}_{K} \mathbf{e}_{K}
\end{array}\right] .
$$

Since $\left(\mathbf{F}_{k} \mathbf{e}_{k}\right)^{T} \mathbf{F}_{k} \mathbf{e}_{k}=\left\|\mathbf{F}_{k} \mathbf{e}_{k}\right\|_{2}^{2}, 1 \leq k \leq K$, from (48) in Appendix 1, we have the bound for $\left\|\mathbf{F}_{k} \mathbf{e}_{k}\right\|_{2}^{2}$, i.e.,

$$
\left(1-\theta-\mu_{1}\right)\left\|\mathbf{e}_{k}\right\|_{2}^{2} \leq\left\|\mathbf{F}_{k} \mathbf{e}_{k}\right\|_{2}^{2} \leq\left(1+\mu_{1}\right)\left\|\mathbf{e}_{k}\right\|_{2}^{2}, \forall k .
$$

Furthermore, because $\left|\left(\mathbf{F}_{k} \mathbf{e}_{k}\right)^{T} \mathbf{F}_{j} \mathbf{e}_{j}\right|=\mid\left\langle\mathbf{F}_{k} \mathbf{e}_{k}, \mathbf{F}_{j} \mathbf{e}_{j}||, 1 \leq\right.$ $k, j \leq K, k \neq j$, from (54) in Appendix 1, we have

$$
\left|\left(\mathbf{F}_{k} \mathbf{e}_{k}\right)^{T} \mathbf{F}_{j} \mathbf{e}_{j}\right| \leq \mu_{2}\left\|\mathbf{e}_{k}\right\|_{2}\left\|\mathbf{e}_{j}\right\|_{2} \leq \mu_{2}\|\mathbf{e}\|_{2}^{2},
$$

where $\|\mathbf{e}\|_{2}=\max _{k}\left\|\mathbf{e}_{k}\right\|_{2}$. Let $d_{k, j}$ denote the $(k, j)$ th entry of $\mathbf{D}$, then $d_{k, j}$ satisfies the following properties:

- $\left(1-\theta-\mu_{1}\right)\|\mathbf{e}\|_{2}^{2} \leq d_{k, k} \leq\left(1+\mu_{1}\right)\|\mathbf{e}\|_{2}^{2}, \forall k$;

- $\sum_{j=1, j \neq k}^{K}\left|d_{k, j}\right| \leq(K-1) \mu_{2}\|\mathbf{e}\|_{2}^{2}, \forall k$.

From Theorem 3 in Appendix 1, we have that

$$
\lambda(\mathbf{D}) \leq\left(1+\mu_{1}+\mu_{2}(K-1)\right)\|\mathbf{e}\|_{2}^{2}=\varphi\|\mathbf{e}\|_{2}^{2},
$$

where $\varphi=1+\mu_{1}+\mu_{2}(K-1)$. This implies that $\lambda$ max $\leq$ $\varphi\|\mathbf{e}\|_{2}^{2}$. Therefore, we have

$$
\left\|\mathbf{B}_{\mathbf{e}} \mathbf{x}\right\|_{2} \leq \sqrt{\varphi}\|\mathbf{e}\|_{2}\|\mathbf{x}\|_{2} .
$$

Insert (34) into (28), we have

$$
\|\mathbf{n}\|_{2} \leq \sqrt{\varphi}\|\mathbf{e}\|_{2}\|\mathbf{x}\|_{2}+\|\mathbf{v}\|_{2} .
$$

Thus, from Theorem 2, we have the coherence-based bound on the performance of the CS-NOMA scheme in the case that CSI is not perfect, i.e.,

$$
\begin{aligned}
\|\mathbf{x}-\hat{\mathbf{x}}\|_{2} & \leq \frac{\sqrt{\varphi}\|\mathbf{e}\|_{2}\|\mathbf{x}\|_{2}+\|\mathbf{v}\|_{2}+\epsilon}{\sqrt{1-\mu_{\mathbf{B}}(4 S-1)}} \\
& \leq \frac{\sqrt{\varphi}\|\mathbf{e}\|_{2}\|\mathbf{x}\|_{2}+\|\mathbf{v}\|_{2}+\epsilon}{\sqrt{1-\mu_{\text {up }}(4 S-1)}},
\end{aligned}
$$

where $S<\left(1 / \mu_{\text {up }}+1\right) / 4$. Furthermore, when $\mu_{1}, \mu_{2} \rightarrow$ 0 , from (36), we have

$$
\|\mathbf{x}-\hat{\mathbf{x}}\|_{2} \leq\|\mathbf{e}\|_{2}\|\mathbf{x}\|_{2}+\|\mathbf{v}\|_{2}+\epsilon,
$$


which is supported by the perfect LCS signatures. Note that (36)-(37) are the results of the worst-case analysis and typically overestimate the actual error.

\section{Simulation results}

In our simulations, we consider an MMTC scenario in which $K$ users sporadically access to a single BS over the multi-path wireless channels in the presence of AWGN. All users share $N$ CDMA chips, i.e., the gain of LCS signature is $N$. We adopt BPSK modulation and assume that all users' wireless channels have $L$ paths. Here, we consider that the entries of the channel response vector $\mathbf{h}_{k}(1 \leq k \leq K)$ follow i.i.d. $\mathcal{N}(0,1)$ for user $k$. Furthermore, we also discuss the case that the CSI is not perfect and assume that the entries of the channel response estimated error vector $\mathbf{e}_{k}(1 \leq k \leq K)$ are i.i.d. Gaussian variables with $\mathcal{N}\left(0, \sigma^{2}\right)\left(\sigma^{2} \ll 1\right)$. In addition, the LCS matrix $\mathcal{F} \in \mathbb{R}^{N \times K}$ is generated by Algorithm 1 with the predetermined parameters $M, \mu_{1}, \mu_{2}$. The OMP $[25,26]$ are employed to demonstrate the effectiveness of the proposed scheme. We evaluate the mean-square-error (MSE) performance, which is defined as

$$
\text { MSE }=\frac{\|\hat{\mathbf{x}}-\mathbf{x}\|_{2}}{\|\mathbf{x}\|_{2}},
$$

where $\hat{\mathbf{x}}$ is an estimate of $\mathbf{x}$.

Based on the analysis in Section 4, we present the MSE performance for the cases that the CSI is perfect and imperfect.

In the case that CSI is perfect, from (25) we have

$$
\frac{\|\mathbf{x}-\hat{\mathbf{x}}\|_{2}}{\|\mathbf{x}\|_{2}} \leq \operatorname{MSE}_{\mu}=\frac{\|\mathbf{v}\|_{2}+\epsilon}{\|\mathbf{x}\|_{2} \sqrt{1-\mu_{\text {up }}(4 S-1)}},
$$

where $S<\left(1 / \mu_{\text {up }}+1\right) / 4$. For the CS-NOMA scheme using the perfect LCS signatures (i.e., $\mu_{\mathbf{B}} \rightarrow 0$ ), we have

$$
\mathrm{MSE}_{1}=\frac{\|\mathbf{v}\|_{2}+\epsilon}{\|\mathbf{x}\|_{2}} .
$$

In the case that CSI is not perfect, from (36) we have

$$
\frac{\|\mathbf{x}-\hat{\mathbf{x}}\|_{2}}{\|\mathbf{x}\|_{2}} \leq \operatorname{MSE}_{\sigma}=\sqrt{\varphi}\|\mathbf{e}\|_{2}+\operatorname{MSE}_{\mu} .
$$

When $\mu_{1}, \mu_{2} \rightarrow 0$, from (37) we have

$$
\mathrm{MSE}_{2}=\|\mathbf{e}\|_{2}+\mathrm{MSE}_{1} \text {. }
$$

Note that (39)-(42) are the worst-case results and typically overestimate the actual MSEs. In addition, we consider $\epsilon=0$ for these MSEs in (39)-(42).

\subsection{Validation for the coherences with different LCS signatures}

For ease of reference, we denote $\mu(\mathbf{B})_{\max }$ as the maximum coherence of different $\mathbf{B}$ that consist of different $\mathcal{H}$ and a fixed $\mathcal{F}$, and $\mu(\hat{\mathbf{B}})_{\max }$ is defined as the maximum coherence of different $\hat{\mathbf{B}}$ which consist of different
$\hat{\mathcal{H}}=\left[\hat{\mathbf{h}}_{1}, \ldots, \hat{\mathbf{h}}_{K}\right]$ and a fixed $\mathcal{F}$. Furthermore, for comparison, we give a counterexample of the LCS matrix $\mathcal{F}$, which is given by

$$
\overline{\mathcal{F}}=[\mathbf{I}, \mathcal{F}],
$$

where $\mathbf{I}$ and $\mathcal{F}$ are the identity matrix and the LCS matrix generated by Algorithm 1, respectively. For the identity matrix I, we have that $\theta=1$ and $\mu_{2}=1$, which implies that the LCS signatures in $\overline{\mathcal{F}}$ do not satisfy the properties (11) and (12) in Theorem 1.

Figure 2 shows $\mu(\mathbf{B})$ versus $\mu_{1}, \mu_{2}$ with $N=M=800$, $\beta=1.5, L=4$. Note that $\mathbf{B}_{\overline{\mathcal{F}}}$ and $\mathbf{B}_{\mathcal{F}}$ respectively represent the cases that consist of $\overline{\mathcal{F}}$ and $\mathcal{F}$, and $\hat{\mathbf{B}}_{\mathcal{F}}$ denotes the matrix $\hat{\mathbf{B}}$ that consists of $\mathcal{F}$ in the imperfect CSI case with $1 / \sigma^{2}=10 \mathrm{~dB}$. From the figure, we see that, for different $\mu_{1}, \mu_{2}$, the plane of $\mu_{\mathrm{up}}$ is higher than the planes of $\mu\left(\mathbf{B}_{\mathcal{F}}\right)_{\max }$ and $\mu\left(\hat{\mathbf{B}}_{\mathcal{F}}\right)_{\max }$, but lower than the plane of $\mu\left(\mathbf{B}_{\overline{\mathcal{F}}}\right)_{\max }$. Thus, when $\mu_{1}$ and $\mu_{2}$ are given (i.e., given $\mathcal{F}$ ), $\mu\left(\mathbf{B}_{\mathcal{F}}\right)_{\max }$ and $\mu\left(\hat{\mathbf{B}}_{\mathcal{F}}\right)_{\text {max }}$ are always less than $\mu_{\text {up }}$, i.e., $\mu_{\text {up }}$ is the upper bound of $\mu(\mathbf{B})$ and $\mu(\hat{\mathbf{B}})$. These results verify that the designed LCS matrix $\mathcal{F}$ can ensure that the coherence of the effective channel matrix, i.e., $\mu(\mathbf{B})$, has an upper bound $\mu_{\text {up }}$ that has nothing to do with the channel response matrix $\mathcal{H}$. Since the differences between $\hat{\mathbf{B}}$ and $\mathbf{B}$ only exist in the channel response matrix, $\mu(\hat{\mathbf{B}})$ and $\mu(\mathbf{B})$ have the same upper bound $\mu_{\mathrm{up}}$. In addition, since the LCS signatures in $\overline{\mathcal{F}}$ do not satisfy Theorem 1 , $\mu\left(\mathbf{B}_{\overline{\mathcal{F}}}\right)_{\max }$ is always equal to 1 for different $\mu_{1}$ and $\mu_{2}$. In summary, these simulation results verify the validity of the designed LCS signatures based on Theorem 1.

\subsection{Performance in perfect CSI case}

Figure 3 shows the MSE versus $\mu_{1}, \mu_{2}$ for different sparsities with $N=M=800, L=4, \beta=1.5$, and SNR $=25 \mathrm{~dB}$. In the figure, the notation MSE $\overline{\mathcal{F}}$ represents the MSE performance of the CS-NOMA scheme using the LCS matrix $\overline{\mathcal{F}}$ in (43) with $\rho_{\mathrm{a}}=1 \%$. Note that the CS-NOMA scheme always adopts the LCS matrix $\mathcal{F}$ satisfying Theorem 1 unless otherwise specified in this paper. It can be seen from the figure that for different $\mu_{1}$ and $\mu_{2}$ (within a certain range, e.g., $0.03 \leq \mu_{1} \leq 0.09$ and $0.26 \leq \mu_{2} \leq 0.32$ ), the MSE performances are almost the same when the sparsity of the signal $\mathbf{x}$ is given. In addition, the MSE performance of the CS-NOMA scheme using the LCS matrix $\mathcal{F}$ is the best when the sparsity of $\mathbf{x}$ is $S_{\text {min }}$, and it decreases with the increase of $\rho_{\mathrm{a}}$; when $\rho_{\mathrm{a}}=6 \%$, it is worse than the $\mathrm{MSE}_{1}$ performance which is achieved by using perfect LCS signatures. As for comparison, the MSE $\_\mathcal{F}$ performance is the worst, which implies that the CS-NOMA scheme using $\overline{\mathcal{F}}$ can not work. 


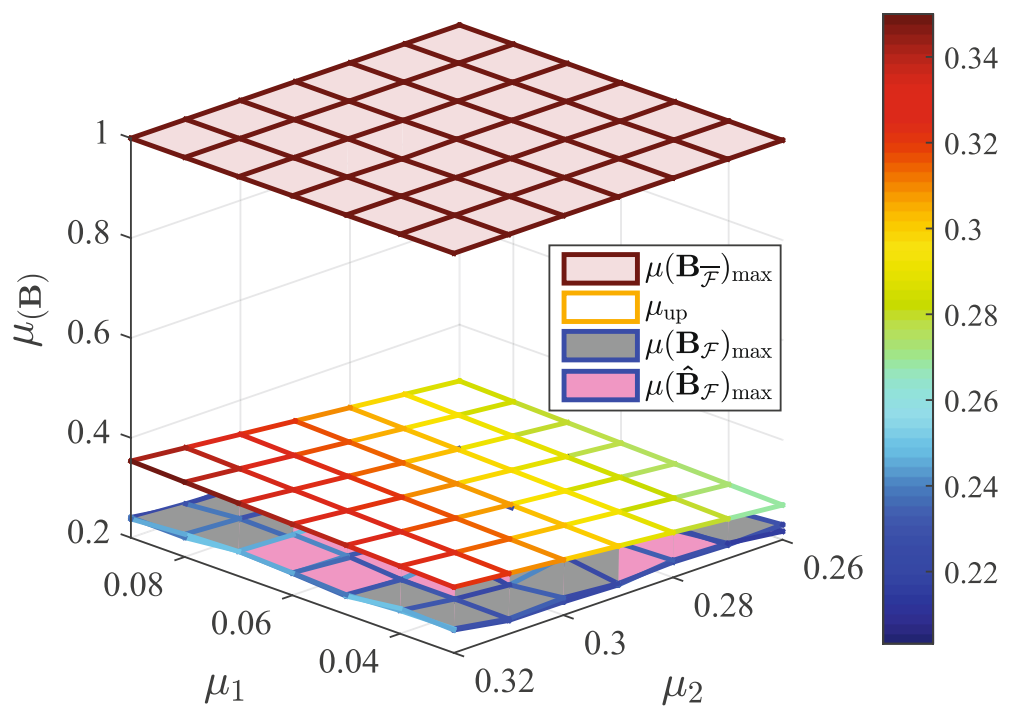

Fig. $2 \mu(\mathbf{B})$ versus $\mu_{1}$ and $\mu_{2}$ with $N=M=800, \beta=1.5$, and $L=4$

Furthermore, we also simulate the MSE versus SNR for different sparsities with $N=M=800, L=4, \beta=1.5$, $\mu_{1}=0.03$, and $\mu_{2}=0.26$, as shown in Fig. 4. It can be seen that the MSE performance of the CS-NOMA scheme with $\mathcal{F}$ improves with the increase of SNR and decreases with the increase of $\rho_{\mathrm{a}}$; when $\rho_{\mathrm{a}} \geq 5 \%$, it is close to the $\mathrm{MSE}_{1}$ performance at high SNR, but always better than the $\mathrm{MSE}_{\mu}$ performance. However, as for comparison, the MSE $\overline{\mathcal{F}}$ performance is almost unchanged and quite poor for different SNR, even worse than both $\mathrm{MSE}_{1}$ and $\mathrm{MSE}_{\mu}$ performances at high SNR.
In Fig. 5, we show the MSE versus SNR for different $\beta$ with $N=M=800, L=4, \rho_{\mathrm{a}}=1 \%, \mu_{1}=0.03$, and $\mu_{2}=0.28$. From the figure, it can be seen that the MSE performance of the CS-NOMA scheme improves with the reduction of $\beta$ at the same conditions. In addition, the MSE performance of the CS-NOMA scheme is always better than the $\mathrm{MSE}_{1}$ performance when $\beta \leq 4$. But, when $\beta=5$, it is worse than the $\mathrm{MSE}_{1}$ performance at high SNR, e.g., $S N R \geq 20 \mathrm{~dB}$. Thus, the proposed scheme can achieve a system overload of 4 when the active users are relatively sparse with an activity ratio of $1 \%$.

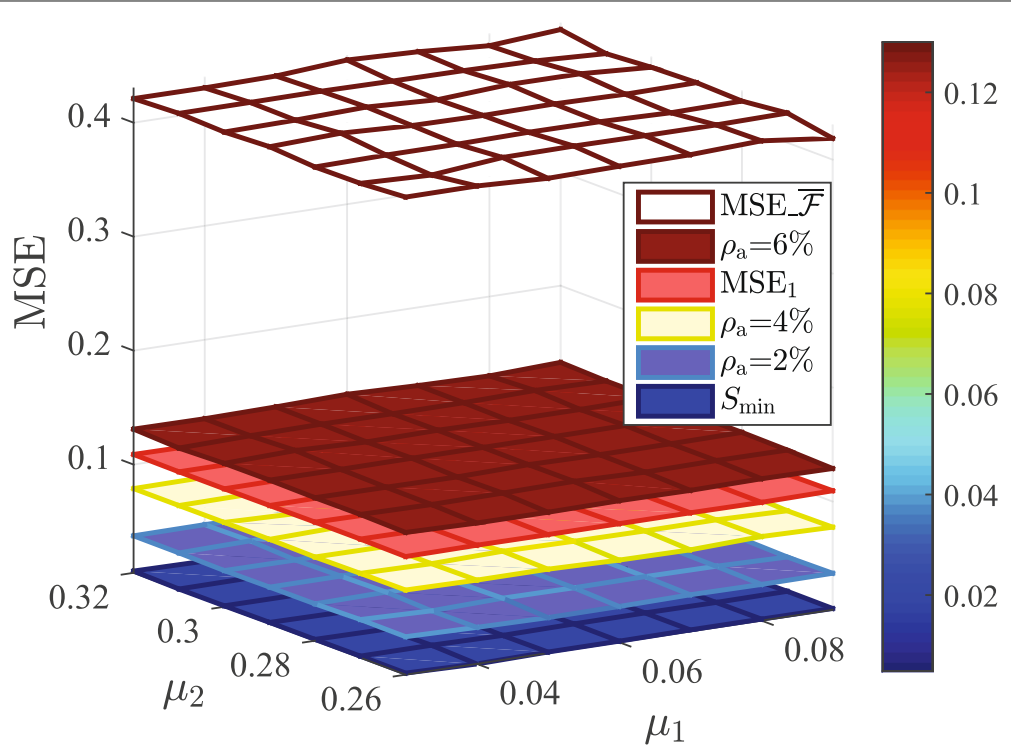

Fig. 3 The MSE versus $\mu_{1}, \mu_{2}$ for different sparsities with $N=M=800, L=4, \beta=1.5$, and SNR $=25 \mathrm{~dB}$ 


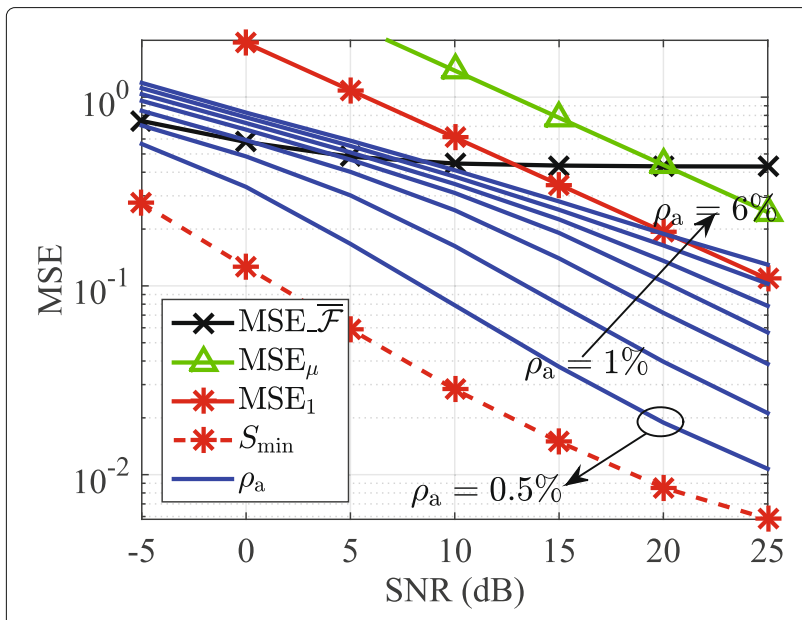

Fig. 4 The MSE versus SNR for different sparsities with $N=M=800$, $L=4, \beta=1.5, \mu_{1}=0.03$, and $\mu_{2}=0.26$

In addition, we also provide the BER performance of the CS-NOMA scheme in Fig. 6 with $N=M=800, L=4$, $\beta=1.5, \mu_{1}=0.03$, and $\mu_{2}=0.26$. In the figure, we compare the BER performances of the CS-NOMA scheme using the LCS signature with that using the PN signature which is the typical spreading sequence in CS-based MTC $[9,13,21,22]$. Here, after the column vector normalization of the PN matrix which contains the PN signatures of all users, we obtain $\theta=L / M=1 / 200, \mu_{1}=0.31$, and $\mu_{2}=0.47$ for the PN matrix with $L=4$, which indicates that the PN matrix satisfies Theorem 1. Therefore, these PN signatures used in the simulation are also LCS signatures, as such the BER performance of the CSNOMA schemes using the LCS signature are almost the same with that using the PN signature at the same sparsity. These results further validate the availability of our proposed scheme.

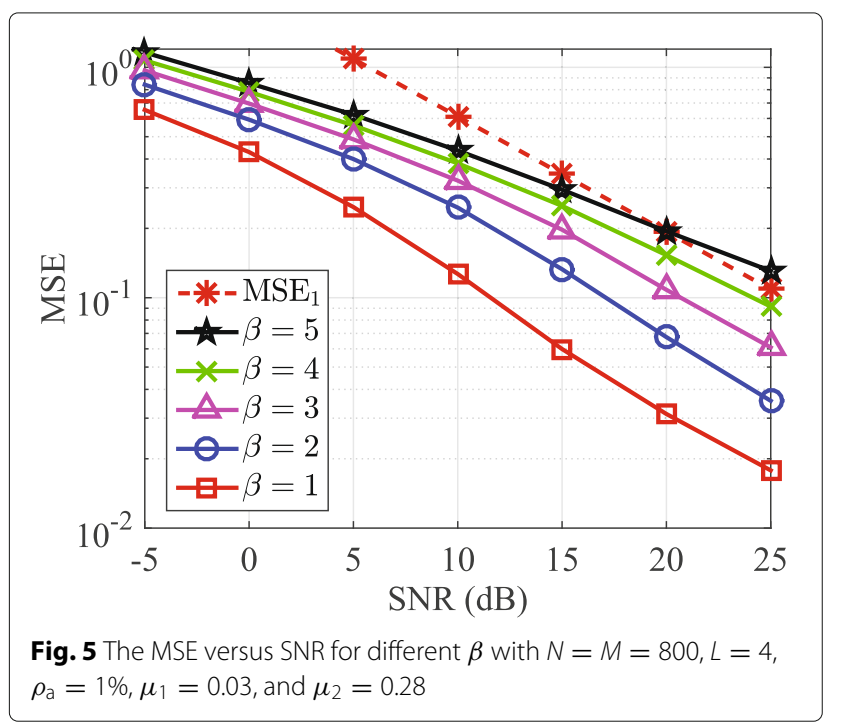

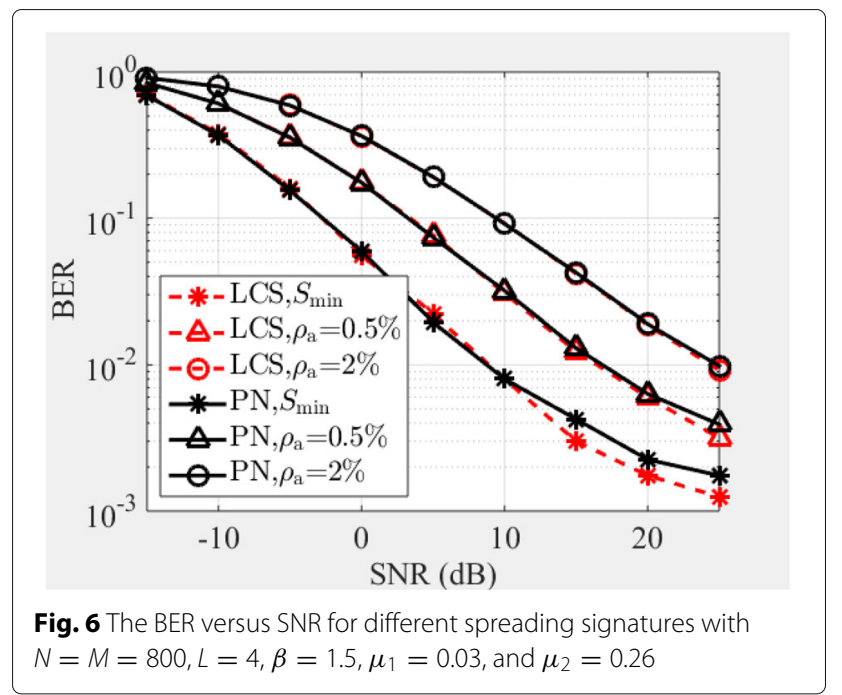

From the simulation results in Figs. 3, 4, 5, and 6, we can see that the designed LCS signatures based on Theorem 1 can provide guarantee for the recovery of at least $S_{\mathrm{min}^{-}}$ sparse signal $\mathbf{x}$. In addition, both $\mathrm{MSE}_{\mu}$ and $\mathrm{MSE}_{1}$ provide bounds for the MSE for the proposed CS-NOMA scheme. It also admits the validity of the proposed CS-NOMA scheme. Furthermore, the proposed scheme achieves a relatively high system overload when the active users are relatively sparse, which implies that the CS-NOMA scheme can significantly improve the spectral efficiency.

\subsection{Performance in imperfect CSI case}

Figure 7 shows the MSE versus $\mu_{1}, \mu_{2}$ in the imperfect CSI case with $N=M=800, \beta=1.5, L=4, \rho_{\mathrm{a}}=1 \%$, and $\mathrm{SNR}=25 \mathrm{~dB}$. In the figure, the notations $15 \mathrm{~dB}$ represents $1 / \sigma^{2}=15 \mathrm{~dB}$. We can see that for different $\mu_{1}, \mu_{2}$ (within a certain range, e.g., $0.03 \leq \mu_{1} \leq 0.09$ and $0.26 \leq$ $\mu_{2} \leq 0.32$ ), the MSE performances are almost the same when $1 / \sigma^{2}$ is given. Additionally, the MSE performance in

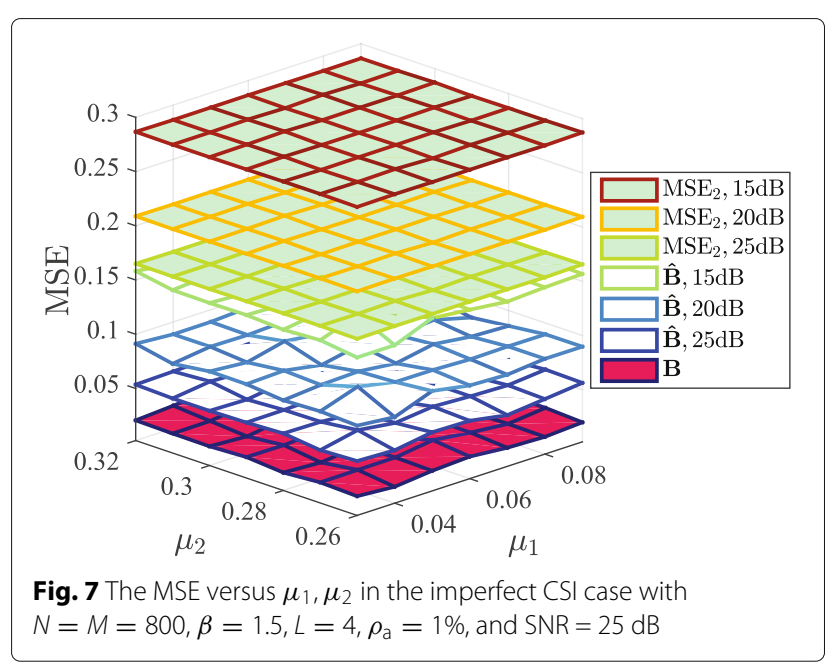




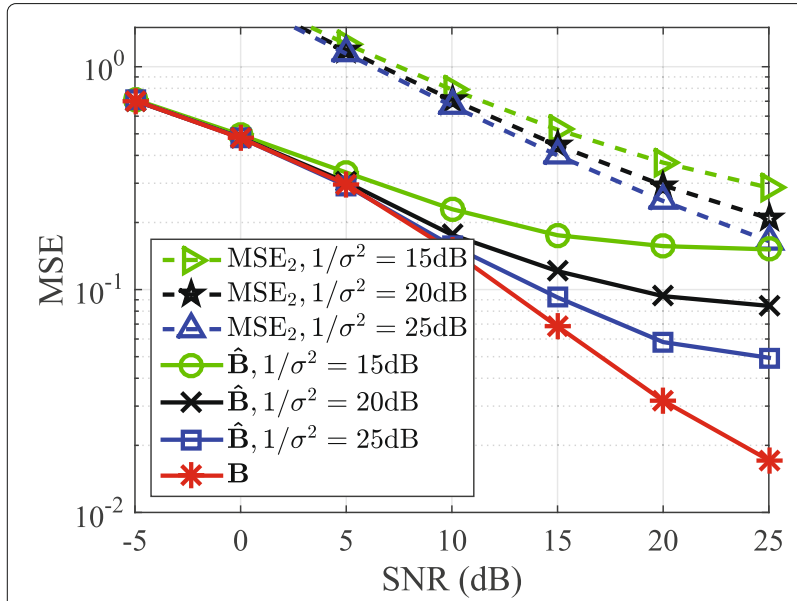

Fig. 8 The MSE versus SNR in the imperfect CSI case with $N=M=800, \beta=1.5, L=4, \rho_{\mathrm{a}}=1 \%, \mu_{1}=0.03$, and $\mu_{2}=0.26$

the imperfect CSI case improves with the increase of $1 / \sigma^{2}$ and is better than the $\mathrm{MSE}_{2}$ performances, but it is worse than the MSE performance in the perfect CSI case with the same conditions. These results imply that even in the imperfect SCI case, the LCS matrix $\mathcal{F}$ ensures that $\mathbf{B}$ is an effective sensing matrix.

In order to analyze the MSE performance in the imperfect CSI case for different SNRs, Fig. 8 shows the MSE versus SNR with $N=M=800, \beta=1.5, L=4, \rho_{\mathrm{a}}=1 \%$, $\mu_{1}=0.03$, and $\mu_{2}=0.26$. From the figure, we can see that the MSE performance improves with the increase of SNR, and it also improves with the increase of $1 / \sigma^{2}$ in the imperfect CSI case. Furthermore, the $\mathrm{MSE}_{2}$ results are better than other MSE results at the same SNR.

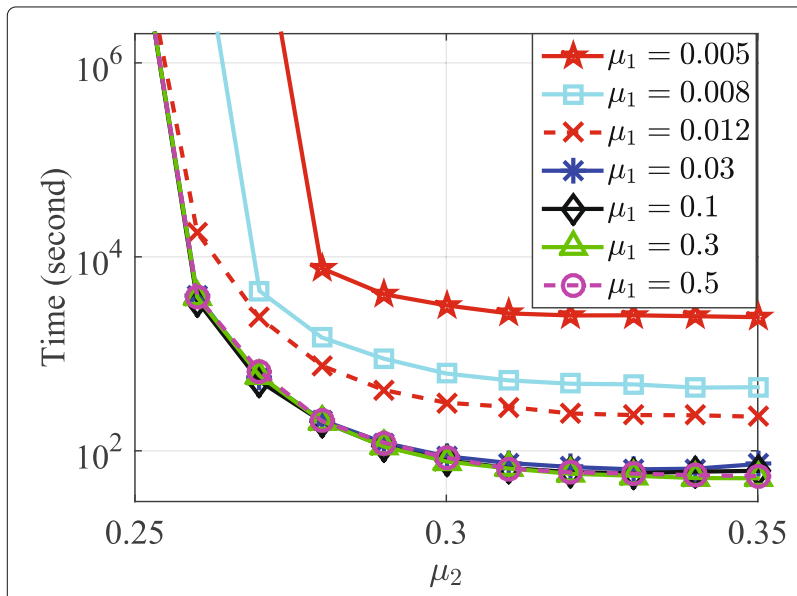

Fig. 9 Simulation time of Algorithm 1 versus $\mu_{2}$ for different $\mu_{1}$ with $N=M=800, L=4$, and $\beta=1.5$
The results in Figs. 7 and 8 imply that the LCS matrix $\mathcal{F}$ designed based on Theorem 1 ensures effective recovery of sparse signal even in the imperfect CSI case. In addition, the proposed CS-NOMA scheme enables joint activity users and data detection through CS-MUD, even if the CSI is not perfect.

\subsection{Convergence and computational complexity of the proposed algorithm}

It is hard to theoretically analyze the convergence performance and the complexity of Algorithm 1; therefore, we use simulation approach to numerically quantify these performances. Figure 9 shows the simulation time of Algorithm 1 versus $\mu_{2}$ for different $\mu_{1}$. From the figure, we can see that when $\mu_{1}$ are fixed, the simulation time slowly increases with decrease of $\mu_{2}$. But it will tend to be infinite when $\mu_{2}$ is below a certain value, e.g., $\mu_{2}<0.26$ for $\mu_{1}>0.03$. This indicates that Algorithm 1 may not be convergent when $\mu_{2}$ is below a certain value. Furthermore, when $\mu_{2}$ is fixed, the simulation time decreases with increase of $\mu_{1}$, but is almost saturated when $\mu_{1}$ is greater than a certain value, e.g., $\mu_{1}>0.03$. And, the convergence time of Algorithm 1 also increases with decrease of $\mu_{1}$. The results imply that the convergence and computational complexity of Algorithm 1 are significantly affected by $\mu_{1}$ and $\mu_{2}$, especially when $\mu_{1}$ and $\mu_{2}$ take low values.

\section{Conclusions}

In this paper, a novel CS-NOMA scheme for MMTC in 5G was proposed to enable joint detection of active users and their data. Due to the introduction of the LCS signature, the proposed CS-NOMA scheme can achieve a relatively high system overload. Then, we presented a theorem for guiding the design of the LCS signatures which provided the theoretical guarantee for ensuring that the CS-MUD could be effectively deployed in the base station. Furthermore, we presented a construction algorithm for the LCS matrix. We also discussed the imperfect CSI case and presented a corresponding bound for the performance of signal recovery for the CS-NOMA scheme. The simulation results shown that the CS-NOMA scheme not only supported massive access, but also had high spectral efficiency and low transmission latency.

\section{Appendix 1}

In order to prove Theorem 1, we first provide Theorem 3 that plays an important role in the proof of Theorem 1.

Theorem 3 (Gershgorin Circle Theorem, Theorem 2 in [39]). The eigenvalues of an $N \times N$ matrix $\mathbf{M}$ with entries $m_{i j}, 1 \leq i, j \leq N$, lie in the union of $N$ discs $d_{i}=d_{i}\left(c_{i}, r_{i}\right)$, $1 \leq i \leq N$, centered at $c_{i}=m_{i i}$ and with radius $r_{i}=$ $\sum_{i \neq j}\left|\bar{m}_{i j}\right|$. 
Now, we prove Theorem 1 in the following.

Proof Based on the definition of coherence for a matrix, $\mu$ (B) can be written as

$$
\mu(\mathbf{B})=\max _{k \neq j} \mu\left(\mathbf{F}_{k} \mathbf{h}_{k}, \mathbf{F}_{j} \mathbf{h}_{j}\right), 1 \leq k, j \leq K .
$$

From (44), in order to obtain the upper bound of $\mu(\mathbf{B})$, we need to analyze the upper bound of $\mu\left(\mathbf{F}_{k} \mathbf{h}_{k}, \mathbf{F}_{j} \mathbf{h}_{j}\right)$ which is given by

$$
\mu\left(\mathbf{F}_{k} \mathbf{h}_{k}, \mathbf{F}_{j} \mathbf{h}_{j}\right)=\frac{\mid\left\langle\mathbf{F}_{k} \mathbf{h}_{k}, \mathbf{F}_{j} \mathbf{h}_{j} \|\right|}{\left\|\mathbf{F}_{k} \mathbf{h}_{k}\right\|_{2}\left\|\mathbf{F}_{j} \mathbf{h}_{j}\right\|_{2}}, k \neq j, \forall k, j .
$$

Therefore, we first analyze the bound of $\left\|\mathbf{F}_{k} \mathbf{h}_{k}\right\|_{2}\left\|\mathbf{F}_{j} \mathbf{h}_{j}\right\|_{2}$ in Step 1. Then the upper bound of $\left|\left\langle\mathbf{F}_{k} \mathbf{h}_{k}, \mathbf{F}_{j} \mathbf{h}_{j}\right\rangle\right|$ will be discussed in Step 2.

Step 1. For arbitrary $k \in\{1, \ldots, K\}$, the bound of $\left\|\mathbf{F}_{k} \mathbf{h}_{k}\right\|_{2}^{2}$ satisfies

$$
\lambda_{\min }^{k}\left\|\mathbf{h}_{k}\right\|_{2}^{2} \leq\left\|\mathbf{F}_{k} \mathbf{h}_{k}\right\|_{2}^{2} \leq \lambda_{\max }^{k}\left\|\mathbf{h}_{k}\right\|_{2}^{2},
$$

where $\lambda_{\min }^{k}$ and $\lambda_{\max }^{k}$ are the minimum and maximum eigenvalues of $\mathbf{G}^{k}=\mathbf{F}_{k}^{\top} \mathbf{F}_{k} \in \mathbb{R}^{L \times L}$, respectively. Let $g_{m, n}^{k}$ denotes the $(m, n)$ th entry of $\mathbf{G}^{k}, 1 \leq m, n \leq L$. Since we have $\sum_{n=1, n \neq m}^{L}\left|\mu_{m, n}^{k k}\right| \leq \mu_{1}, \forall m$, and $\sum_{l=1}^{L-1} f_{N-l+1, k}^{2} \leq \theta, \forall k$, then $g_{m, n}^{k}$ satisfies the following properties:

- $1-\theta \leq g_{m, m}^{k} \leq 1,1 \leq m \leq L, \forall k$;

- $\sum_{n=1, n \neq m}^{L}\left|g_{m, n}^{k}\right|=\sum_{n=1, n \neq m}^{L}\left|\mu_{m, n}^{k k}\right| \leq \mu_{1}, \forall m, k$.

From Theorem 3, all the eigenvalues of $\mathbf{G}^{k}$ satisfy the following inequality

$$
1-\theta-\mu_{1} \leq \lambda\left(\mathbf{G}^{k}\right) \leq 1+\mu_{1}, \forall k,
$$

where $\lambda$ (A) represents the eigenvalues of $\mathbf{A}$. This implies that $1-\theta-\mu_{1} \leq \lambda_{\min }^{k}<\lambda_{\max }^{k} \leq 1+\mu_{1}$. Thus, for arbitrary $k$, from (46), the bound of $\left\|\mathbf{F}_{k} \mathbf{h}_{k}\right\|_{2}^{2}$ is given by

$$
\left(1-\theta-\mu_{1}\right)\left\|\mathbf{h}_{k}\right\|_{2}^{2} \leq\left\|\mathbf{F}_{k} \mathbf{h}_{k}\right\|_{2}^{2} \leq\left(1+\mu_{1}\right)\left\|\mathbf{h}_{k}\right\|_{2}^{2}, \forall k .
$$

Therefore, we have the bound of $\left\|\mathbf{F}_{k} \mathbf{h}_{k}\right\|_{2}\left\|\mathbf{F}_{j} \mathbf{h}_{j}\right\|_{2}$, i.e.,

$$
\begin{aligned}
& \left(1-\theta-\mu_{1}\right)\left\|\mathbf{h}_{k}\right\|_{2}\left\|\mathbf{h}_{j}\right\|_{2} \leq\left\|\mathbf{F}_{k} \mathbf{h}_{k}\right\|_{2}\left\|\mathbf{F}_{j} \mathbf{h}_{j}\right\|_{2} \\
& \leq\left(1+\mu_{1}\right)\left\|\mathbf{h}_{k}\right\|_{2}\left\|\mathbf{h}_{j}\right\|_{2}, \forall k, j .
\end{aligned}
$$

In addition, since all columns of $\mathbf{F}_{k}$ are linearly independent (i.e., $\mathbf{F}_{k}$ is full rank), $\mathbf{G}^{k}$ is a Gram matrix which means that $\mathbf{G}^{k}$ is positive definite. Thus, from Theorem 3 , the inequality $\theta+\mu_{1}<1$ is necessary to be held.

Step 2. Now, we discuss the upper bound of $\left|\left\langle\mathbf{F}_{k} \mathbf{h}_{k}, \mathbf{F}_{j} \mathbf{h}_{j}\right\rangle\right|$ which can be rewritten as $\left|\left\langle\mathbf{h}_{k}, \mathbf{F}_{k}^{\top} \mathbf{F}_{j} \mathbf{h}_{j}\right\rangle\right|$, then we have

$$
\left|\left\langle\mathbf{F}_{k} \mathbf{h}_{k}, \mathbf{F}_{j} \mathbf{h}_{j}\right\rangle\right| \leq\left\|\mathbf{h}_{k}\right\|_{2}\left\|\mathbf{F}_{k}^{\top} \mathbf{F}_{j} \mathbf{h}_{j}\right\|_{2} \leq \sqrt{\lambda_{\max }^{k j}}\left\|\mathbf{h}_{k}\right\|_{2}\left\|\mathbf{h}_{j}\right\|_{2},
$$

where $\lambda_{\max }^{k j}$ represents the maximum eigenvalue of $\mathbf{P}^{k j}=$ $\left(\mathbf{F}_{k}^{\top} \mathbf{F}_{j}\right)^{\top} \mathbf{F}_{k}^{\top} \mathbf{F}_{j} \in \mathbb{R}^{L \times L}$. Let $p_{t, r}^{k j}$ denotes the $(t, r)$ th entry of $\mathbf{P}^{k j}, 1 \leq t, r \leq L$, then $p_{t, t}^{k j}=\sum_{l=1}^{L}\left(\mu_{l, t}^{k j}\right)^{2} \geq 0$ and $p_{t, r}^{k j}=$ $\sum_{l=1}^{L} \mu_{l, t}^{k j} \mu_{l, r}^{k j} \leq \sum_{l=1}^{L}\left|\mu_{l, t}^{k j}\right|\left|\mu_{l, r}^{k j}\right|, r \neq t$. Let $\sum_{t}$ denotes the sum of the absolute values of elements in $t$-row of $\mathbf{P}^{k j}$, i.e.,

$$
\sum_{t}=\sum_{r=1}^{L}\left|p_{t, r}^{k j}\right| \leq \sum_{r=1}^{L} \sum_{l=1}^{L}\left|\mu_{l, t}^{k j}\right|\left|\mu_{l, r}^{k j}\right| .
$$

From Theorem 3, we know that $\lambda_{\max }^{k j} \leq \min _{t} \sum_{t}$. Furthermore, from the root-mean square-arithmetic meangeometric mean (RMS-AM-GM) inequality [40], we have

$$
\sum_{l=1}^{L}\left|\mu_{l, t}^{k j}\right|\left|\mu_{l, r}^{k j}\right| \leq\left(\sum_{l=1}^{L} a_{l}\right)^{2} / L
$$

when $a_{1}=a_{2}=\cdots=a_{L}$, where $a_{l}=$ $\left(\left|\mu_{l, t}^{k j}\right|+\left|\mu_{l, r}^{k j}\right|\right) / 2$. Since $\sum_{l=1}^{L}\left|\mu_{l, t}^{k j}\right| \leq \mu_{2}$ for arbitrary $t$, (52) is rewritten as $\sum_{l=1}^{L}\left|\mu_{l, t}^{k j}\right|\left|\mu_{l, r}^{k j}\right| \leq \mu_{2}^{2} / L, \forall t, r$. Then, we have

$$
\sum_{t} \leq \sum_{r=1}^{L} \sum_{l=1}^{L}\left|\mu_{l, t}^{k j}\right|\left|\mu_{l, r}^{k j}\right| \leq \mu_{2}^{2}, \forall t,
$$

which implies $\lambda_{\max }^{k j} \leq \mu_{2}^{2}$. From (50), the upper bound of $\left|\left\langle\mathbf{F}_{k} \mathbf{h}_{k}, \mathbf{F}_{j} \mathbf{h}_{j}\right\rangle\right|$ is given by

$$
\left|\left\langle\mathbf{F}_{k} \mathbf{h}_{k}, \mathbf{F}_{j} \mathbf{h}_{j}\right\rangle\right| \leq \mu_{2}\left\|\mathbf{h}_{k}\right\|_{2}\left\|\mathbf{h}_{j}\right\|_{2}, k \neq j, \forall k, j .
$$

Therefore, combining (49) and (54), we have the upper bound of $\mu\left(\mathbf{F}_{k} \mathbf{h}_{k}, \mathbf{F}_{j} \mathbf{h}_{j}\right)$ for arbitrary $k, j, k \neq j$, i.e.,

$$
\mu\left(\mathbf{F}_{k} \mathbf{h}_{k}, \mathbf{F}_{j} \mathbf{h}_{j}\right) \leq \frac{\mu_{2}}{1-\theta-\mu_{1}}, k \neq j, \forall k, j,
$$

which implies that $\mu(\mathbf{B}) \leq \frac{\mu_{2}}{1-\theta-\mu_{1}}$. Thus, we complete the proof of Theorem 1 .

\section{Appendix 2}

Now we prove Corollary 1 using the similar tricks in the proof of Theorem 1 as follows.

Proof Let $\Lambda$ be an arbitrary subset of $\{1, \ldots, K\}$ and $|\Lambda|=S$, then $\mathbf{B}_{\Lambda} \in \mathbb{R}^{N \times S}$ is obtained by only keeping the columns of $\mathbf{B}$ corresponding to $\Lambda$. Similar to this, $\mathcal{H}_{\Lambda}$ and $\mathcal{F}_{\Lambda}$ are obtained by only keeping the columns of $\mathcal{H}$ and $\mathcal{F}$ corresponding to $\Lambda$, respectively. Thus, $\mathbf{B}_{\Lambda}$ is generated by $\mathcal{H}_{\Lambda}$ and $\mathcal{F}_{\Lambda}$. Let $\mathbf{h}_{s}$ and $\mathbf{f}_{s}$ be the $s$-column 
$(1 \leq s \leq S)$ of $\mathcal{H}_{\Lambda}$ and $\mathcal{F}_{\Lambda}$, respectively. Accordingly, $\mathbf{F}_{s}$ is generated by $\mathbf{f}_{s}$ in the form of (8). Hence, we have that $\mathbf{B}_{\Lambda}=\left[\mathbf{F}_{1} \mathbf{h}_{1}, \ldots, \mathbf{F}_{S} \mathbf{h}_{S}\right]$. Now, we analyze the bound of the eigenvalues of $\mathbf{C}=\mathbf{B}_{\Lambda}^{\top} \mathbf{B}_{\Lambda} \in \mathbb{R}^{S \times S}$.

Let $c_{i, s}$ be the $(i, s)$ th entry of $\mathbf{C}, 1 \leq i, s \leq S$, then $c_{i, i}=\left\|\mathbf{F}_{i} \mathbf{h}_{i}\right\|_{2}^{2}$ and $c_{i, s}=\left\langle\mathbf{F}_{i} \mathbf{h}_{i}, \mathbf{F}_{s} \mathbf{h}_{s}\right\rangle, i \neq s$. From (48) and (54), and recall $\left\|\mathbf{h}_{k}\right\|_{2}^{2}=1, \forall k$, the entries of $\mathbf{C}$ satisfy the following properties:

- $1-\theta-\mu_{1} \leq c_{i, i} \leq 1+\mu_{1}, 1 \leq i \leq S$;

- $\left|c_{i, s}\right| \leq \mu_{2}, i \neq s, 1 \leq i, s \leq S$.

Then, $\sum_{s=1, s \neq i}^{S}\left|c_{i, s}\right| \leq(S-1) \mu_{2}, 1 \leq i \leq S$, from Theorem 3, we have

$$
1-\theta-\mu_{1}-(S-1) \mu_{2} \leq \lambda(\mathbf{C}) \leq 1+\mu_{1}+(S-1) \mu_{2},
$$

which implies that

$$
\left(1-\delta_{S}\right)\|\mathbf{x}\|_{2}^{2} \leq\left\|\mathbf{B}_{\Lambda} \mathbf{x}\right\|_{2}^{2}<\left(1+\delta_{S}\right)\|\mathbf{x}\|_{2}^{2},
$$

where $\delta_{S}=\theta+\mu_{1}+(S-1) \mu_{2}$ and $\mathbf{x} \in \mathbb{R}^{S \times 1}$. Note that Theorem 3 needs the inequality $1-\delta_{S}>0$ to be held, which implies $S<1+\left(1-\theta-\mu_{1}\right) / \mu_{2}$, i.e., $S<1+$ $1 / \mu_{\text {up }}$. In addition, because (57) holds for arbitrary $\Lambda \in$ $\{1, \ldots, K\}$ with $|\Lambda|=S$, so $\left(1-\delta_{S}\right)\|\mathbf{x}\|_{2}^{2} \leq\|\mathbf{B x}\|_{2}^{2}<$ $\left(1+\delta_{S}\right)\|\mathbf{x}\|_{2}^{2}$, where $\mathbf{x} \in \mathbb{R}^{K \times 1}$ is an $S$-sparse signal. Thus, we complete the proof.

\section{Acknowledgements}

This work is supported in part by the National Natural Science Foundation of China under Grants 61671086 and 61629101, the 111 Project (NO.B17007), and the Director Funds of Beijing Key Laboratory of Network System Architecture and Convergence (NO.2017BKL-NSAC-ZJ-04).

\section{Funding}

Not applicable.

\section{Availability of data and materials}

Not applicable.

\section{Authors' contributions}

The authors have contributed jointly to all parts of the preparation of this manuscript, and all authors read and approved the final manuscript.

\section{Authors' information}

Not applicable.

\section{Competing interests}

The authors declare that they have no competing interests.

\section{Publisher's Note}

Springer Nature remains neutral with regard to jurisdictional claims in published maps and institutional affiliations.

Received: 14 November 2017 Accepted: 5 March 2018 Published online: 19 April 2018

\section{References}

1. 3GPP, Service requirements for machine-type communications (MTC). Rel. 12, 2-27 (2013)

2. JG Andrews, S Buzzi, W Choi, SV Hanly, A Lozano, AC Soong, JC Zhang, What will 5 G be?. IEEE J Sel. Areas Commun. 32(6), 1065-1082 (2014)
3. N Alliance, 5 G white paper. Next Gener. Mob. Netw. White Pap (2015). https://www.ngmn.org/5g-white-paper/5g-white-paper.html

4. J-P Hong, W Choi, BD Rao, Sparsity controlled random multiple access with compressed sensing. IEEE Trans. Wirel. Commun. 14(2), 998-1010 (2015)

5. M Hasan, E Hossain, D Niyato, Random access for machine-to-machine communication in LTE-advanced networks: Issues and approaches. IEEE Commun. Mag. 51(6), 86-93 (2013)

6. DL Donoho, Compressed sensing. IEEE Trans. Inf. Theory. 52(4), 1289-1306 (2006)

7. EJ Candès, T Tao, Near-optimal signal recovery from random projections: Universal encoding strategies?. IEEE Trans. Inf. Theory. 52(12), 5406-5425 (2006)

8. W Chen, MR Rodrigues, IJ Wassell, Projection design for statistical compressive sensing: A tight frame based approach. IEEE Trans. Signal Process. 61(8), 2016-2029 (2013)

9. C Bockelmann, HF Schepker, A Dekorsy, Compressive sensing based multi-user detection for machine-to-machine communication. Trans. Emerg. Telecommun. Technol. 24(4), 389-400 (2013)

10. Y Beyene, C Boyd, K Ruttik, C Bockelmann, O Tirkkonen, R Jäntti, in AFRICON, 2015. Compressive sensing for MTC in new LTE uplink multi-user random access channel (IEEE, Addis Ababa, 2015), pp. 1-5

11. F Monsees, M Woltering, C Bockelmann, A Dekorsy, in 2015 IEEE 81st Vehicular Technology Conference (VTC Spring). Compressive sensing multi-user detection for multicarrier systems in sporadic machine type communication (IEEE, Glasgow, 2015), pp. 1-5

12. C Bockelmann, F Monsees, M Woltering, A Dekorsy, Hardware-in-the-loop measurements of the multi-carrier compressed sensing multi-user detection (mcsm) system. arxiv preprint arxiv:1511.09278 (2015)

13. HF Schepker, A Dekorsy, in 2011 8th International Symposium on Wireless Communication Systems (ISWCS). Sparse multi-user detection for cdma transmission using greedy algorithms (IEEE, Aachen, 2011), pp. 291-295

14. Y Saito, Y Kishiyama, A Benjebbour, T Nakamura, A Li, K Higuchi, in 2013 IEEE 77th Vehicular Technology Conference (VTC Spring). Non-orthogonal multiple access (NOMA) for cellular future radio access (IEEE, Dresden, 2013), pp. 1-5

15. R Hoshyar, FP Wathan, R Tafazolli, Novel low-density signature for synchronous cdma systems over awgn channel. IEEE Trans. Signal Proc. 56(4), 1616-1626 (2008)

16. M Al-Imari, P Xiao, MA Imran, R Tafazolli, in 2014 11th International Symposium on Wireless Communications Systems (ISWCS). Uplink non-orthogonal multiple access for $5 \mathrm{~g}$ wireless networks (IEEE, Barcelona, 2014), pp. 781-785

17. H Nikopour, H Baligh, in 2013 IEEE 24th International Symposium on Personal Indoor and Mobile Radio Communications (PIMRC). Sparse code multiple access (IEEE, London, 2013), pp. 332-336

18. Z Yuan, G Yu, W Li, Multi-user shared access for $5 \mathrm{~g}$. Telecommun. Netw. Technol. 5(5), 28-30 (2015)

19. Z Ding, X Lei, GK Karagiannidis, R Schober, J Yuan, VK Bhargava, A survey on non-orthogonal multiple access for $5 \mathrm{~g}$ networks: Research challenges and future trends. IEEE J. Sel. Areas Commun. 35(10), 2181-2195 (2017)

20. L Dai, B Wang, Y Yuan, S Han, I Chih-Lin, Z Wang, Non-orthogonal multiple access for $5 \mathrm{G}$ : Solutions, challenges, opportunities, and future research trends. IEEE Commun. Mag. 53(9), 74-81 (2015)

21. B Wang, L Dai, T Mir, Z Wang, Joint user activity and data detection based on structured compressive sensing for noma. IEEE Commun. Lett. 20(7), 1473-1476 (2016)

22. B Wang, L Dai, Y Zhang, T Mir, J Li, Dynamic compressive sensing-based multi-user detection for uplink grant-free noma. IEEE Commun. Lett. 20(11), 2320-2323 (2016)

23. K He, Y Li, C Yin, in 2016 IEEE 27th Annual International Symposium on Personal, Indoor, and Mobile Radio Communications (PIMRC). Sparse multi-user detection for non-orthogonal multiple access in 5G systems (IEEE, Valencia, 2016), pp. 1-5

24. L Applebaum, WU Bajwa, MF Duarte, R Calderbank, Asynchronous code-division random access using convex optimization. Phys. Commun. $\mathbf{5}(2), 129-147(2012)$

25. YC Pati, R Rezaiifar, PS Krishnaprasad, in 1993 Conference Record of The Twenty-Seventh Asilomar Conference on Signals, Systems and Computers. Orthogonal matching pursuit: Recursive function approximation with applications to wavelet decomposition (IEEE, Pacific Grove, 1993), pp. 40-44 
26. JA Tropp, AC Gilbert, Signal recovery from random measurements via orthogonal matching pursuit. IEEE Trans. Inf. Theory. 53(12), 4655-4666 (2007)

27. MF Duarte, YC Eldar, Structured compressed sensing: From theory to applications. IEEE Trans. Signal Process. 59(9), 4053-4085 (2011)

28. DL Donoho, M Elad, Optimally sparse representation in general (nonorthogonal) dictionaries via $\ell_{1}$ minimization. Proc. Natl. Acad. Sci. 100(5), 2197-2202 (2003)

29. DL Donoho, X Huo, Uncertainty principles and ideal atomic decomposition. EEE Trans. Inf. Theory. 47(7), 2845-2862 (2001)

30. JA Tropp, Greed is good: Algorithmic results for sparse approximation. EEE Trans. Inf. Theory. 50(10), 2231-2242 (2004)

31. R Gribonval, M Nielsen, Sparse representations in unions of bases. EEE Trans. Inf. Theory. 49(12), 3320-3325 (2003)

32. L Welch, Lower bounds on the maximum cross correlation of signals. EEE Trans. Inf. Theory. 20(3), 397-399 (1974)

33. T Strohmer, RW Heath, Grassmannian frames with applications to coding and communication. Appl. Comput. Harmon. Anal. 14(3), 257-275 (2003)

34. EJ Candès, T Tao, Decoding by linear programming. IEEE Trans. Inf. Theory. 51(12), 4203-4215 (2005)

35. MA Davenport, Random observations on random observations: Sparse signal acquisition and processing. PhD thesis, Rice University (2010)

36. DL Donoho, M Elad, VN Temlyakov, Stable recovery of sparse overcomplete representations in the presence of noise. IEEE Trans. Inf. Theory. 52(1), 6-18 (2006)

37. MA Davenport, MF Duarte, YC Eldar, G Kutyniok, Introduction to compressed sensing. Preprint. 93(1), 2 (2011)

38. N Bourbaki, Topological Vector Spaces: Chapters 1-5. (Springer Science \& Business Media, France, 2013)

39. SA Geršhgorin, Über die abgrenzung der eigenwerte einer matrix. Akad. Nauk SSSR Ser. Fiz.-Mat. (6), 749-754 (1931)

40. PS Bullen, DS Mitrinovic, M Vasic, Means and Their Inequalities, vol. 31 (Springer Science \& Business Media, Dordrecht, 2013)

\section{Submit your manuscript to a SpringerOpen ${ }^{\circ}$ journal and benefit from:}

- Convenient online submission

- Rigorous peer review

- Open access: articles freely available online

- High visibility within the field

- Retaining the copyright to your article

Submit your next manuscript at $>$ springeropen.com 